\title{
Hydroxyselenylation and Tethered Silanoxyselenylation of Allylic
}

\section{Silanols}

Harshit Joshi ${ }^{\mathrm{a}}$ and Shyam Sathyamoorthi ${ }^{\mathrm{a}, *}$

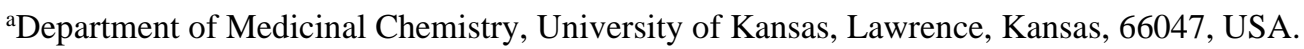

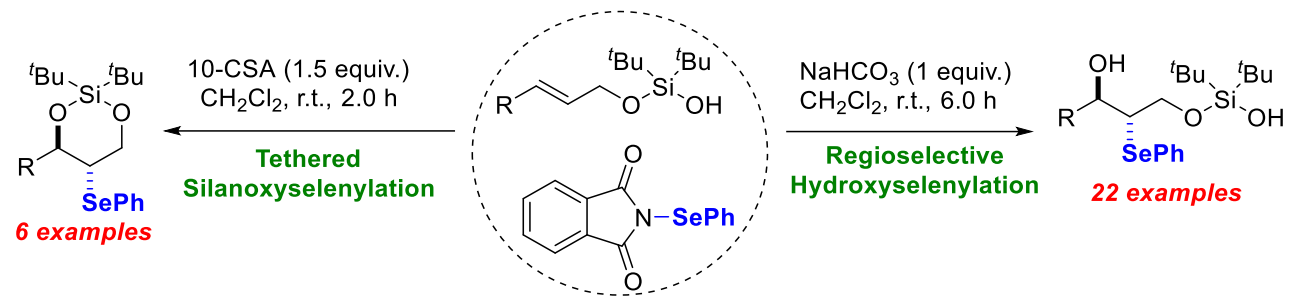

\begin{abstract}
We present protocols for the highly regioselective hydroxyselenylation and silanoxyselenylation of allylic silanols. $N$-(Phenylseleno)phthalimide acts as the selenylating agent for both transformations. Under basic conditions, hydroxyselenylation proceeds with $>20: 1$ regioselectivity, and the products are valuable synthons for further transformations. We show that the silanol plays a critical role in maintaining the yield and regioselectivity of this reaction. Surprisingly, under acidic conditions, the hydroxyselenylation pathway is blocked, and products of a tethered silanoxyselenylation are exclusive.
\end{abstract}

The electrophilic functionalization of unactivated alkenes is an important synthetic approach for the installation of carbon-heteroatom linkages. ${ }^{1}$ Generally, the first step in most of these reactions is the formation of a cyclic three-membered cationic intermediate, which is subsequently opened by a nucleophile. ${ }^{2}$ In such processes, controlling the regioselectivity of nucleophilic attack is often a challenge. "Tethering" a desired nucleophile to an existing functional group in the molecule of interest allows for intramolecular attack, which often proceeds with predictable regioselectivity and high diastereocontrol. ${ }^{3-10}$ Our laboratory has provided the first examples of the use of silanol tethers for the intramolecular ring-opening of both transient and stable electrophiles. ${ }^{11-14}$

Organoselenides have many attractive properties that make them complementary to organomercurials and organohalides. ${ }^{15-17}$ Unlike organohalides, organoselenides are stable to most common synthetic reactions, including hydrolysis, nucleophilic substitution, reduction, and Swern-type oxidations. Organoselenides are also unreactive in 
the presence of nucleophilic nitrogen centers which are often present in heteroarenes of medicinal chemistry importance, and selenylation reagents are highly chemoselective for alkene functionalizations. Furthermore, much like $\mathrm{C}-\mathrm{Hg}$ bonds, which can be facilely cleaved into $\mathrm{C} \cdot \mathrm{C}-\mathrm{Se}$ bonds are excellent radical precursors, ${ }^{18}$ and their reduced toxicity makes organoselenides more attractive intermediates relative to organomercurials (Scheme 1). We were thus interested in developing a tethered silanoxyselenylation of allylic alcohols. Such a reaction would form protected 1,3-diol organoselenides in a single step from the corresponding allylic silanol starting materials. Based on precedent from selenolactonization ${ }^{19,20}$ and selenoetherfication chemistry, ${ }^{21-23}$ we envisioned that such products would be valuable intermediates in the syntheses of important carbohydrate or polyketide targets.

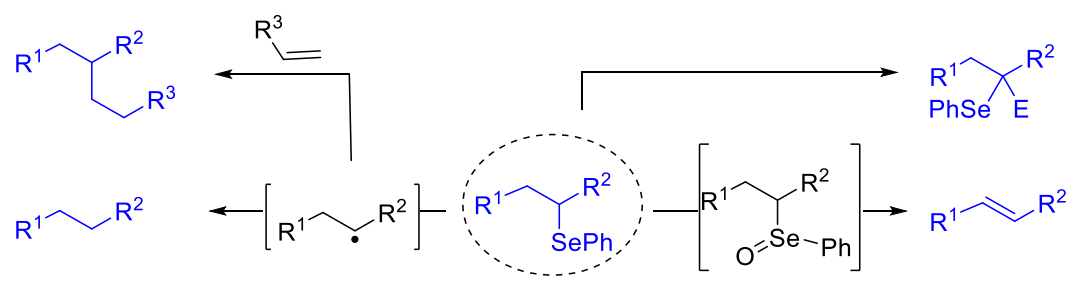

Scheme 1. Prior art has established that organoselenides are versatile synthons.

Table 1. Optimization of a tethered silanoxyselenylation reaction.

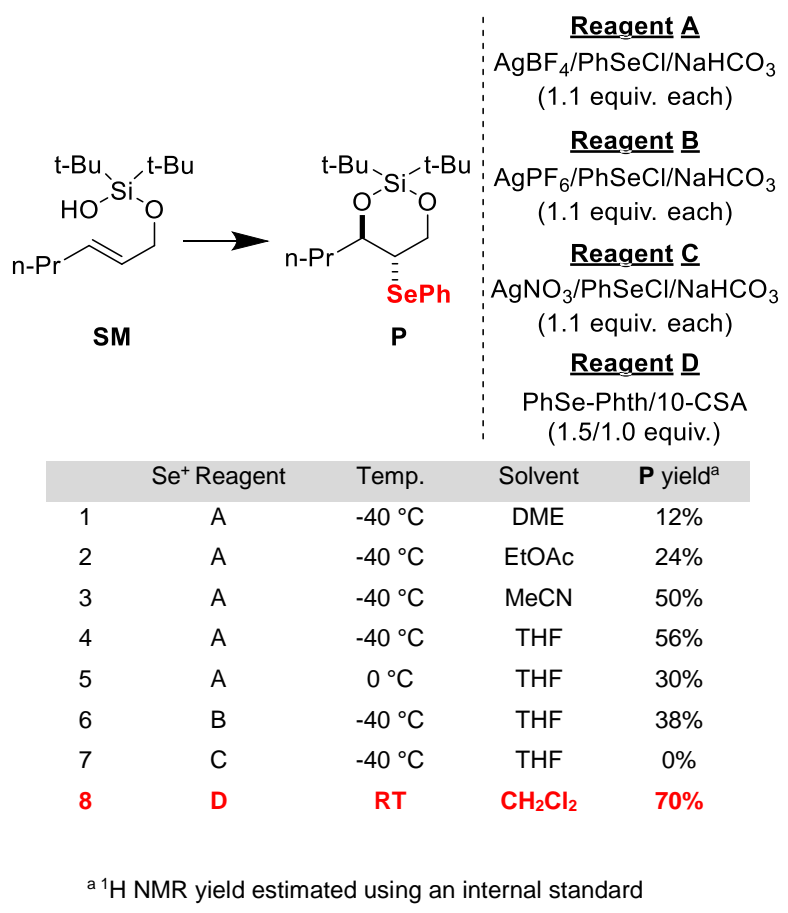

Optimization of this reaction began with (E)-di-tert-butyl(hex-2-en-1-yloxy)silanol, prepared in one step from condensation of commercially available di-tert-butylsilyl bis(trifluoromethanesulfonate) with trans-2-hexen-1- 
ol (Table 1). ${ }^{13}$ With $\mathrm{AgBF}_{4} / \mathrm{PhSeCl}^{24,25} 12 \%$ of product formed in dimethoxyethane (Table 1, Entry 1). Product yield increased when the solvent was switched to ethyl acetate, acetonitrile, and tetrahydrofuran (Table 1, Entries 2-4). In THF, warming the reaction temperature from $-40{ }^{\circ} \mathrm{C}$ to $0{ }^{\circ} \mathrm{C}$ (Table 1, Entry 5) was markedly deleterious. Using $\mathrm{AgPF}_{6}$ (Table 1, Entry 6) or $\mathrm{AgNO}_{3}$ (Table 1, Entry 7) in place of $\mathrm{AgBF}_{4}$ was also harmful. Abandoning silver salts completely and using Nicolaou's $N$-(phenylseleno)phthalimide ${ }^{26,27}$ reagent with 10 -camphorsulfonic acid increased both the product yield and the reaction reproducibility (Table 1, Entry 8).

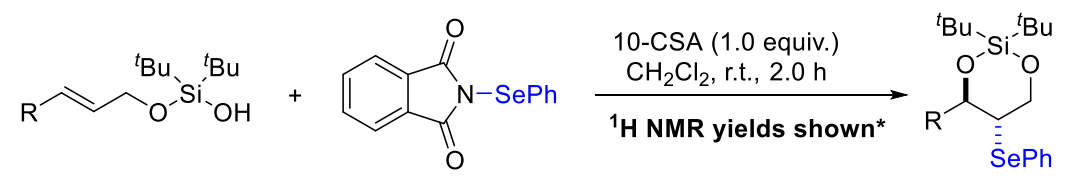

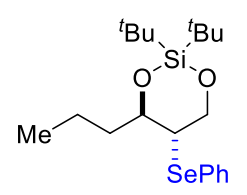

$2 \mathrm{a}, 78 \%(0.1 \mathrm{mmol})$

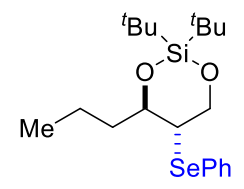

$2 \mathrm{a}, 85 \%$ (1 $\mathrm{mmol})$ 10-fold increase

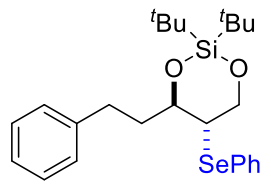

$2 d, 60 \%$

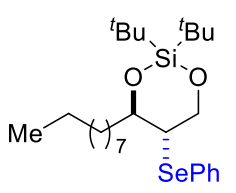

$2 \mathrm{~b}, \mathbf{8 1 \%}$

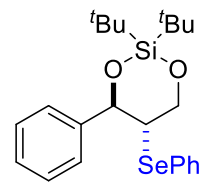

$2 e, 85 \%$

*Compounds are unstable to chromatographic purification.

Scheme 2. Substrate scope of a tethered silanoxyselenylation reaction.

Our optimized protocol was compatible with several allylic silanols (Scheme 2). The reaction was also scalable 10-fold from $0.1 \mathrm{mmol}$ to $1 \mathrm{mmol}$ without loss of yield or selectivity (Scheme 2, Compound 2a). Unfortunately, in all cases examined, the product organoselenide heterocycles were unstable to chromatographic purification with unadulterated silica gel, triethylamine-treated silica gel, neutral alumina, Florisil, and Davisil. In general, starting material was fully consumed in these reactions with few side products. The main impurities were unreacted $N$-(phenylseleno)phthalimide reagent, phthalimide, and 10-camphorsulfonic acid. In the instances shown here (Scheme 2), washing with $0.5 \mathrm{M}$ aqueous $\mathrm{NaOH}$ solution was sufficient to yield reasonably pure compound, but the inability to purify by chromatography hampered our substrate survey.

Over the course of optimization (Table 1), a small amount of hydroxyselenylated product formed in select reactions, and this compound was stable to silica gel chromatography. We thus wondered if we could optimize the 
formation of this product (Scheme 3). Absent a tether, we were unsure whether perfect regiocontrol could be achieved with such a reaction. Amazingly, a simple replacement of 10-camphorsulfonic acid with 1 equivalent of $\mathrm{NaHCO}_{3}$ led to a complete reversal of product selectivity, now exclusively favoring hydroxyselenylated compound. Importantly, this product was a single regioisomer, stable to chromatographic purification, and easily isolable in pure form.

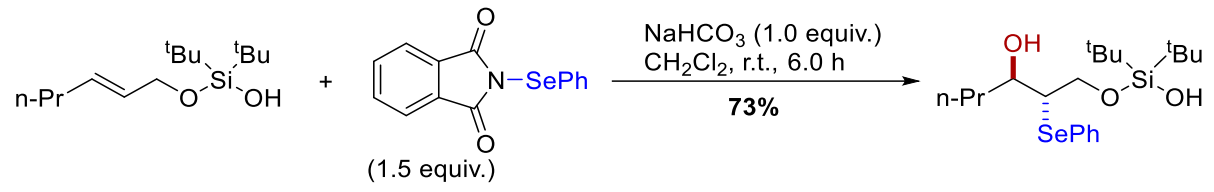

Scheme 3. A pH switch leads to a complete reversal of product selectivity!

This protocol was compatible with a variety of allylic silanols (Scheme 4). In all cases, reactions proceeded, with >20:1 regiocontrol. Our protocol tolerated substrates with branched alkyl chains (Scheme 4, Compounds $\mathbf{4 b - c}$ and $\mathbf{4 j - k}$ ), substituted aryl rings (Scheme 4, Compounds 4d-i, 4p, 4t), heteroaryl rings (Scheme 4, Compounds 4l4m), and aliphatic ethers (Scheme 4, Compounds 4n-4o). We were not constrained to using only trans-allylic silanol starting materials. Cis-allylic silanols reacted smoothly as well to form syn-selenohydrin silanol products (Scheme 4, Compounds $4 \mathbf{u}-\mathbf{v})$. Most of these investigations were conducted using primary allylic silanols. With a secondary allylic silanol, selenohydrin product (Scheme 4, Compound 4s) was formed as a single regioisomer and diastereomer in $40 \%$ yield; $48 \%$ starting material was recovered in the reaction. The relative configuration of the three stereocenters in this molecule was stablished by single crystal X-ray diffraction analysis (CCDC: 2109848). What accounts for the high diastereoselectivity in this case? We propose a model shown in Scheme 5, which suggests that the avoidance of unfavorable steric clashing between the seleniranium adduct and the bulky di-tert-butyl-silanol group may be a primary factor. 


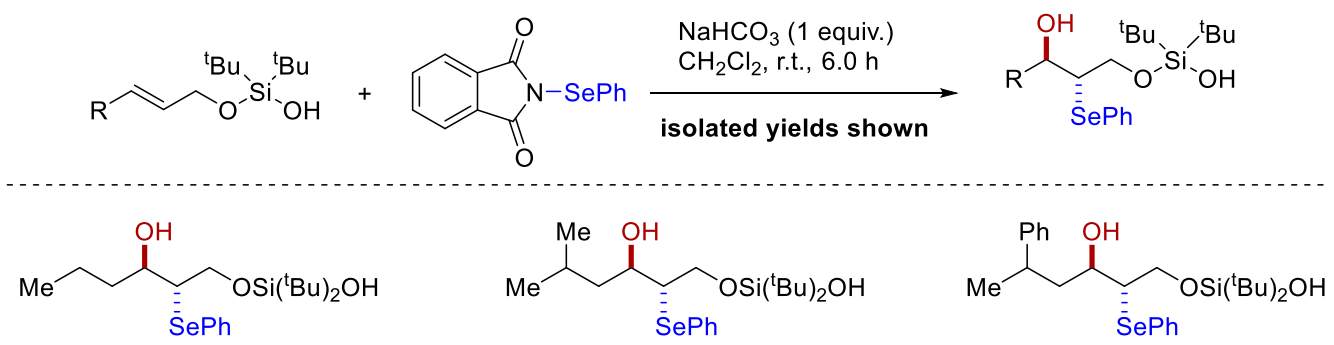

$4 a, 73 \%$

4b, $78 \%$

$4 c, 61 \%$<smiles>[X]c1ccc(CCC(O)[C@H](COCCO)[C@@H]2Oc3ccccc32)cc1</smiles>

4d: $X=H, 69 \%$

4e: $X=p$-Fluoro, $67 \%$

4f: $\mathrm{X}=p$-Chloro, $\mathbf{7 0 \%}$

4g: $X=p$-Bromo, $71 \%$

4h: $\mathrm{X}=p$-lodo, $\mathbf{7 1 \%}$

4i: $X=m$-Trifluoromethyl, $60 \%$

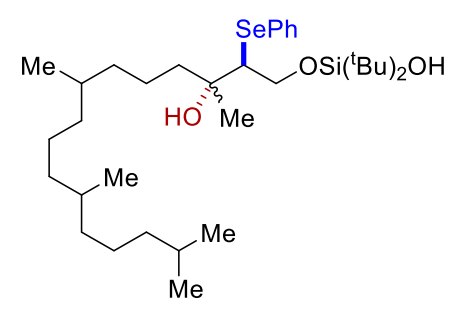

$\overbrace{\mathrm{Me}}^{\mathrm{Se}} \overbrace{\mathrm{S}_{\mathrm{SePh}}}^{\mathrm{OH}} \mathrm{OSi}\left({ }^{\mathrm{t} B u}\right)_{2} \mathrm{OH}$

$4 k, 64 \%$

4j, $30 \%$<smiles>CC(C)(C)OC[C@H](O)C(O)CCc1cc2ccccc2s1</smiles>

$41,68 \%$<smiles>CC(C)(C)OC[C@H]([SeH]c1ccccc1)C(O)CCc1cccs1</smiles>

$4 \mathrm{~m}, 38 \%$<smiles>[14CH3]OC[C@H](O)C(O)CCCOC(=O)c1ccccc1</smiles>

$4 n, 72 \%$

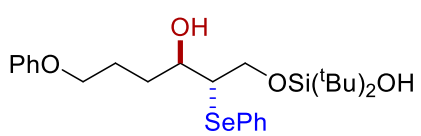

$40,76 \%$<smiles>COc1ccccc1C(O)[C@@H](CO[Si](C)(C)CO)[Se]c1ccccc1</smiles>

$4 p, 72 \%$

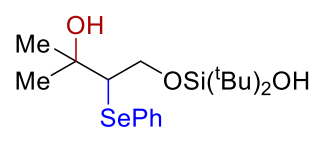

$4 q, 78 \%$

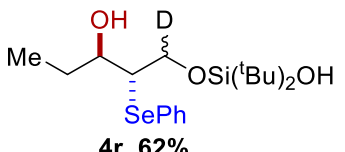

$4 r, 62 \%$<smiles>COc1ccc(CCCC(O)[C@H](CO[SiH3])[SeH2]c2ccccc2)cc1</smiles>

$4 t, 67 \%$

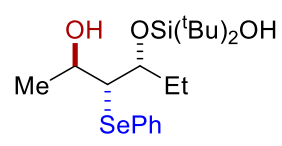

4s, $40 \%$ (48\% RSM)

CCDC: 2109848

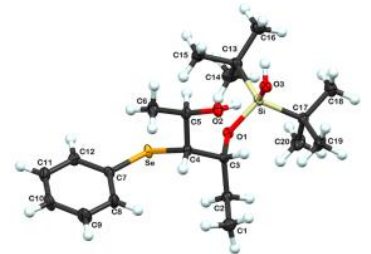

Scheme 4. Substrate scope of our hydroxyselenylation of allylic silanols.
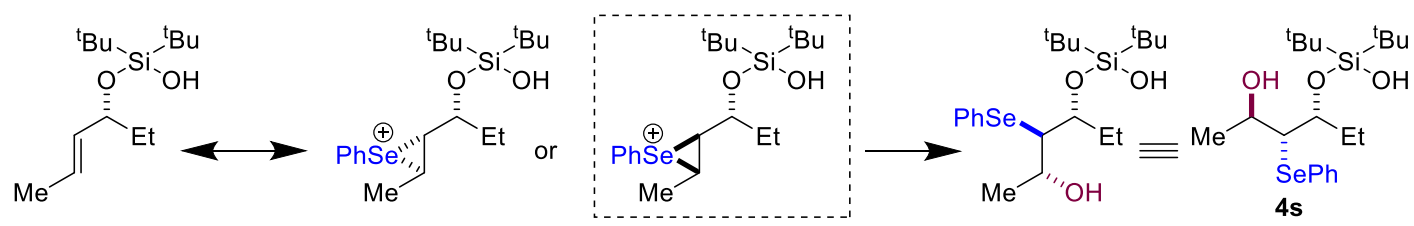

favored

Scheme 5. Avoidance of steric clashing may be a driving force for stereoselectivity. 
What is the role of the silanol auxiliary in this hydroxyselenylation? To answer this question, we conducted a series of control experiments (Scheme 6). With (E)-tert-butyl(hex-2-en-1-yloxy)dimethylsilane, regioisomeric selenohydrin products 5 and $\mathbf{6}$ formed in a combined yield of 35\% (Scheme 6A). With trans-2-hexen-1-ol, selenohydrin product 7 formed in >20:1 regioselectivity and in 50\% yield (Scheme 6B); this yield is $20 \%$ lower than with the corresponding silanol starting material (Scheme 4, Compound 4a). Thus, a hydroxyl group is essential for regiocontrol in this and related reactions, ${ }^{28-30}$ but a free alcohol leads to a drop in yield. Overall, we conclude that the silanol auxiliary serves as a unique protecting group, capable of maintaining $>20: 1$ regioselectivity in the reaction due to presence of the $\mathrm{Si}-\mathrm{OH}$.

A.

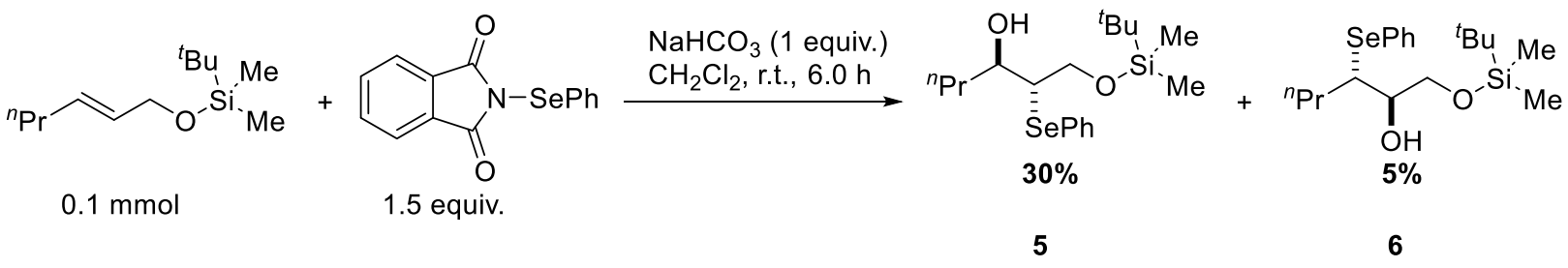

B.

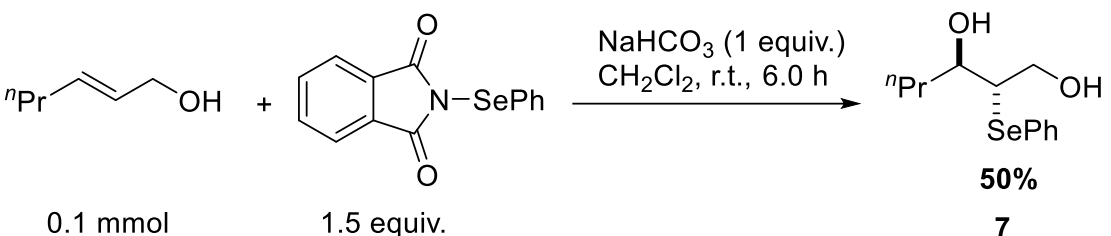

Scheme 6. The di-tert-butylsilanol auxiliary appears to have beneficial effects on both the yield and the regioselectivity of our hydroxyselenylation reaction.

The selenohydrin silanols are versatile synthons (Scheme 7). Oxidation of 4a with Dess-Martin periodinane formed ketone $\mathbf{8}$ in $52 \%$ yield (Scheme 7A). Using acetic anhydride, the $\mathrm{C}-\mathrm{OH}$ rather than the $\mathrm{Si}-\mathrm{OH}$ is preferentially transformed into the corresponding acetate (Scheme 7B). We note that 9 (Scheme 7B) contains differentially protected primary and secondary alcohols, a marked advantage of using allylic silanols as starting materials over the analogous allylic alcohols. 
A.

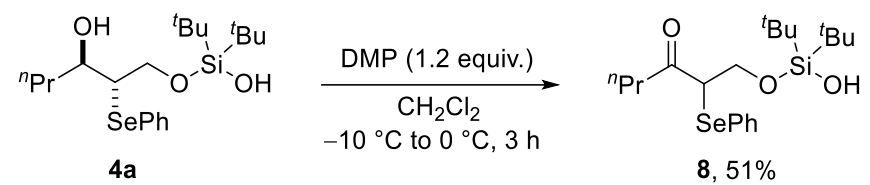

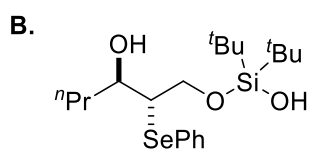

$4 a$

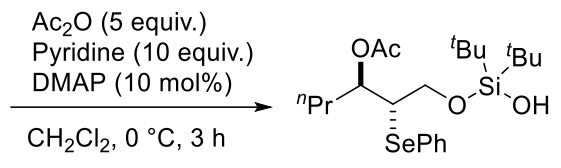

$9,96 \%$

Scheme 7. Selenohydrin silanols are versatile synthons.

In summary, we have developed hydroxyselenylation and silanoxyselenylation reactions of allylic silanols. $N$-(Phenylseleno)phthalimide acts as the selenylating agent for both transformations. Under basic conditions, hydroxyselenylation proceeds with $>20: 1$ regioselectivity, and the products are valuable synthons for further transformations. The silanol plays a critical role in maintaining the yield and regioselectivity of this reaction. Surprisingly, under acidic conditions, the hydroxyselenylation pathway is blocked, and products of a tethered silanoxyselenylation are exclusive. While this latter reaction interesting from a mechanistic standpoint, the products are unfortunately unstable to chromatography on silica gel and alumina. Given the known versatility of organoselenium compounds, we expect the hydroxyselenylation of allylic silanols to be employed in the pursuit of complex, polyfunctional targets of value.

\section{Experimental Section}

\section{General Considerations}

All reagents were obtained commercially unless otherwise noted. Solvents were purified by passage under $10 \mathrm{psi} \mathrm{N}_{2}$ through activated alumina columns. Infrared (IR) spectra were recorded on a Thermo Scientific Nicolet iS5 FT-IR spectrometer; data are reported in frequency of absorption $\left(\mathrm{cm}^{-1}\right)$. NMR data are recorded as chemical shift in ppm referenced internally using residue solvent peaks, multiplicity $(\mathrm{s}=$ singlet, $\mathrm{d}=$ doublet, $\mathrm{t}=$ triplet, $\mathrm{q}=$ quartet, $\mathrm{m}=$ multiplet or overlap of nonequivalent resonances), integration, and coupling constant (Hz). ${ }^{1} \mathrm{H}$ NMR spectra were recorded at 400, 500, or $600 \mathrm{MHz} .{ }^{13} \mathrm{C}$ NMR spectra were recorded at 100 or $125 \mathrm{MHz}$. Exact mass spectra were recorded using an electrospray ion source (ESI) either in positive mode or negative mode with a time-of-flight (TOF) analyzer on a Waters LCT PremierTM mass spectrometer and are given in m/z. TLC was performed on precoated glass plates (Merck) and visualized either with a UV lamp (254 nm) or by dipping into a solution of $\mathrm{KMnO}_{4}-\mathrm{K}_{2} \mathrm{CO}_{3}$ 
in water followed by heating. Flash chromatography was performed on silica gel (230-400 mesh) or Florisil (60-100 mesh).

\section{Allylic Silanol Starting Materials}

Note: Allylic silanol starting material compounds were prepared according to a previously reported procedure. ${ }^{13}$

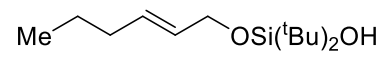

$1 \mathrm{a}$

(E)-di-tert-butyl(hex-2-en-1-yloxy)silanol

Compound 1a: Previously characterized in Org. Lett. 2020, 22, 8665-8669.

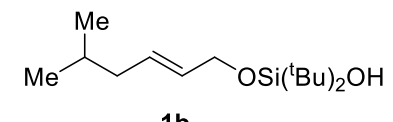

1b

(E)-di-tert-butyl((5-methylhex-2-en-1-yl)oxy)silanol

Compound 1b: Previously characterized in Org. Chem. Front., 2021, 8, 5361-5368.

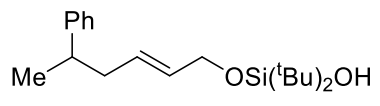

$1 c$

(E)-di-tert-butyl((5-phenylhex-2-en-1-yl)oxy)silanol

Compound 1c: Purified using a gradient of 0 to $0.2 \%$ acetone/DCM on silica gel; (colorless oil, $279 \mathrm{mg}$, $42 \%$ yield); ${ }^{1} \mathrm{H}$ NMR (400 MHz, CDCl $) \delta 7.39-7.30(\mathrm{~m}, 2 \mathrm{H}), 7.30-7.17(\mathrm{~m}, 3 \mathrm{H}), 5.63(\mathrm{~m}, 2 \mathrm{H}), 4.31(\mathrm{~d}, J=3.6 \mathrm{~Hz}, 2 \mathrm{H}), 2.83$ (h, $J=7.0 \mathrm{~Hz}, 1 \mathrm{H}), 2.48-2.38(\mathrm{~m}, 1 \mathrm{H}), 2.38-2.26(\mathrm{~m}, 1 \mathrm{H}), 1.31$ (d, $J=6.9 \mathrm{~Hz}, 3 \mathrm{H}), 1.06$ (s, 9H), 1.05 (s, 9H). ${ }^{13} \mathrm{C}\{1 \mathrm{H}\} \mathrm{NMR}\left(100 \mathrm{MHz}, \mathrm{CDCl}_{3}\right) \delta 147.0,130.9,129.1,128.3,127.0,125.9,64.0,41.0,40.0,27.4,21.6,20.4$. IR $3488,2860,1473,1101,970 \mathrm{~cm}^{-1}$.; HRMS (APCI) m/z: [M-H] calculated for $\mathrm{C}_{20} \mathrm{H}_{33} \mathrm{O}_{2} \mathrm{Si}$ 333.2236, found 333.2255.

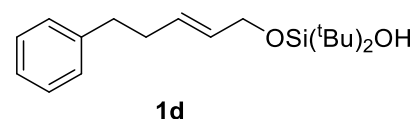

(E)-di-tert-butyl((5-phenylpent-2-en-1-yl)oxy)silanol

Compound 1d: Previously characterized in Org. Lett. 2020, 22, 8665-8669.

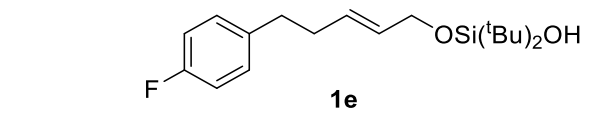

(E)-di-tert-butyl((5-(4-fluorophenyl)pent-2-en-1-yl)oxy)silanol

Compound 1e: Previously characterized in Org. Chem. Front., 2021, 8, 5361-5368. 


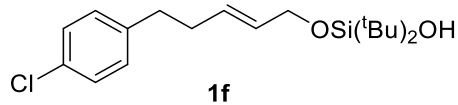

(E)-di-tert-butyl((5-(4-chlorophenyl)pent-2-en-1-yl)oxy)silanol

Compound 1f: Previously characterized in Org. Chem. Front., 2021, 8, 5361-5368.

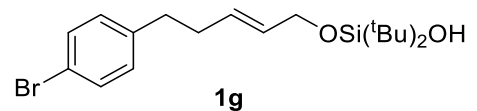

(E)-((5-(4-bromophenyl)pent-2-en-1-yl)oxy)di-tert-butylsilanol

Compound 1g: Previously characterized in Org. Chem. Front., 2021, 8, 5361-5368.

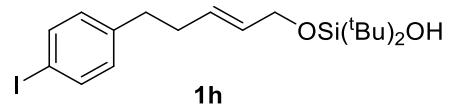

(E)-di-tert-butyl((5-(4-iodophenyl)pent-2-en-1-yl)oxy)silanol

Compound 1h: Previously characterized in Org. Chem. Front., 2021, 8, 5361-5368.

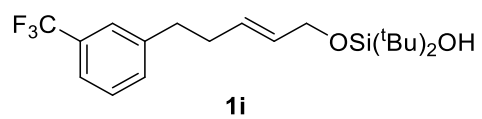

(E)-di-tert-butyl((5-(3-(trifluoromethyl)phenyl)pent-2-en-1-yl)oxy)silanol

Compound 1i: Purified using a gradient of 0 to $0.2 \%$ acetone/DCM on silica gel; (colorless oil, $358 \mathrm{mg}$, $46 \%$ yield); ${ }^{1} \mathrm{H}$ NMR $\left(400 \mathrm{MHz}, \mathrm{CDCl}_{3}\right) \delta 7.48-7.29(\mathrm{~m}, 4 \mathrm{H}), 5.70(\mathrm{dtt}, J=15.6,6.4,1.3 \mathrm{~Hz}, 1 \mathrm{H}), 5.65-5.53(\mathrm{~m}, 1 \mathrm{H}), 4.29$ $(\mathrm{dt}, J=4.9,1.4 \mathrm{~Hz}, 2 \mathrm{H}), 2.76(\mathrm{dd}, J=8.7,6.8 \mathrm{~Hz}, 2 \mathrm{H}), 2.39(\mathrm{tdt}, J=9.2,7.9,1.4 \mathrm{~Hz}, 2 \mathrm{H}), 1.02(\mathrm{~d}, J=1.1 \mathrm{~Hz}$, 18H). ${ }^{13} \mathrm{C}$ NMR (101 MHz, $\left.\mathrm{CDCl}_{3}\right) \delta 142.8,132.0(\mathrm{~d}, J=1.7 \mathrm{~Hz}), 130.8(\mathrm{q}, J=20 \mathrm{~Hz}), 130.6,129.2,128.8,125.8$ (q, $J=270 \mathrm{~Hz}), 125.2(\mathrm{q}, J=3.8 \mathrm{~Hz}), 122.8$ (q, $J=3.9 \mathrm{~Hz}), 63.9,35.5,33.7,27.5,20.6$. IR 3511, 2934, 2860, 1473, 1324, 1130, $833 \mathrm{~cm}^{-1}$; HRMS (APCI) m/z: [M-H] calculated for $\mathrm{C}_{20} \mathrm{H}_{30} \mathrm{~F}_{3} \mathrm{O}_{2} \mathrm{Si}$ 387.1967, Found 387.1955.

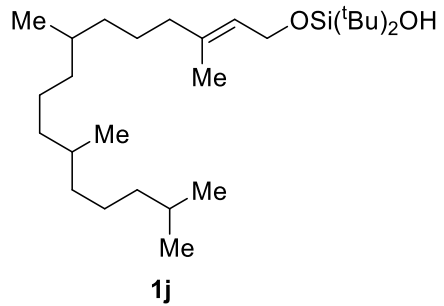

(E)-di-tert-butyl((3,7,11,15-tetramethylhexadec-2-en-1-yl)oxy)silanol

Compound 1j: Previously characterized in J. Org. Chem. 2021, 86, 9233-9243. 


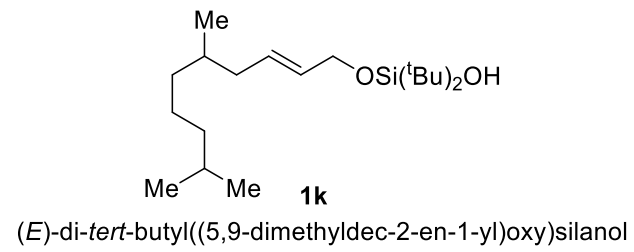

Compound 1k: Previously characterized in Molecules 2021, 26, 3829.

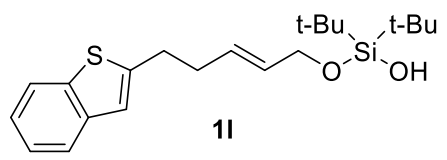

(E)-((5-(benzo[b]thiophen-2-yl)pent-2-en-1-yl)oxy)di-tert-butylsilanol

Compound 11: Purified using a gradient of 0 to $20 \%$ Ethyl acetate/Hexanes on silica gel; (light yellow semi-solid, $225 \mathrm{mg}, 60 \%$ yield); ${ }^{1} \mathrm{H} \mathrm{NMR}\left(400 \mathrm{MHz}, \mathrm{CDCl}_{3}\right) \delta 7.78(\mathrm{dq}, J=7.8,0.9 \mathrm{~Hz}, 1 \mathrm{H}), 7.73-7.62(\mathrm{~m}, 1 \mathrm{H}), 7.36-7.22$ $(\mathrm{m}, 2 \mathrm{H}), 7.04(\mathrm{q}, J=1.0 \mathrm{~Hz}, 1 \mathrm{H}), 5.85-5.73(\mathrm{~m}, 1 \mathrm{H}), 5.73-5.59(\mathrm{~m}, 1 \mathrm{H}), 4.33(\mathrm{dq}, J=5.0,1.3 \mathrm{~Hz}, 2 \mathrm{H}), 3.09-$ 2.95 (m, 2H), 2.53 (tdd, $J=7.5,6.3,1.2 \mathrm{~Hz}, 2 \mathrm{H}), 1.03$ (s, 18H). ${ }^{13} \mathrm{C}\{1 \mathrm{H}\}$ NMR $\left(100 \mathrm{MHz}, \mathrm{CDCl}_{3}\right) \delta 145.6,140.1$, 139.3, 130.7, 129.0, 124.0, 123.4, 122.7, 122.1, 120.7, 63.9, 33.5, 30.6, 27.4, 20.4. IR 3460, 2934, 2854, 1473, 827 $\mathrm{cm}^{-1}$; HRMS (APCI) m/z: [M-H] calculated for $\mathrm{C}_{21} \mathrm{H}_{31} \mathrm{O}_{2} \mathrm{SSi} 375.1820$, found 375.1802 .

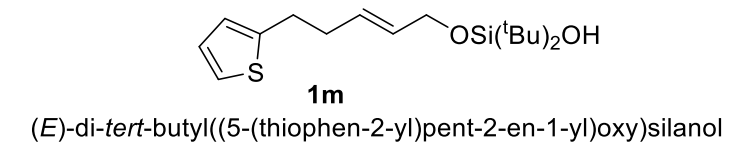

Compound 1m: Purified using a gradient of 0.5 to $5 \%$ diethyl ether/hexane on silica gel; (colorless oil, $411.7 \mathrm{mg}$, $42 \%$ yield). ${ }^{1} \mathrm{H}$ NMR $\left(400 \mathrm{MHz}, \mathrm{CDCl}_{3}\right) \delta 7.11(\mathrm{dd}, J=5.1,1.2 \mathrm{~Hz}, 1 \mathrm{H}), 6.91(\mathrm{dd}, J=5.1,3.4 \mathrm{~Hz}, 1 \mathrm{H}), 6.82-6.75$ (m, 1H), $5.78-5.68(\mathrm{~m}, 1 \mathrm{H}), 5.68-5.58(\mathrm{~m}, 1 \mathrm{H}), 4.35-4.24(\mathrm{~m}, 2 \mathrm{H}), 2.92(\mathrm{t}, J=7.4 \mathrm{~Hz}, 2 \mathrm{H}), 2.53-2.34(\mathrm{~m}, 2 \mathrm{H})$, 1.02 (s, 18H). ${ }^{13} \mathrm{C}\left\{{ }^{1} \mathrm{H}\right\}$ NMR (100 MHz, $\left.\mathrm{CDCl}_{3}\right) \delta 144.8,130.6,129.4,126.8,124.3,123.1,64.0,34.3,29.8,27.5$, 20.6. IR 3648, 2934, 2894, 2860, 1473, 1381, 1113, 1056, 970, 827, 690, $645 \mathrm{~cm}^{-1}$; HRMS (APCI) m/z: [M-H] calculated for $\mathrm{C}_{17} \mathrm{H}_{29} \mathrm{O}_{2} \mathrm{SSi} 325.1663$, found 325.1665.

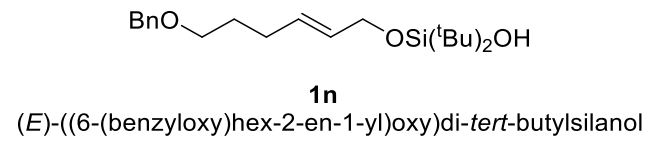

Compound 1n: Previously characterized in Org. Chem. Front., 2021, 8, 5361-5368.

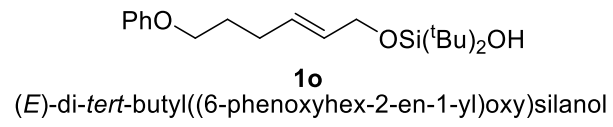

Compound 1o: Previously characterized in Org. Chem. Front., 2021, 8, 5361-5368. 


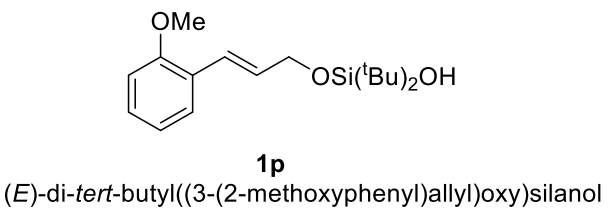

Compound 1p: Previously characterized in Org. Lett. 2020, 22, 8665-8669.

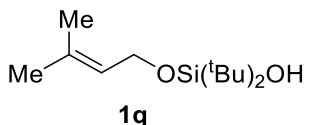

di-tert-butyl((3-methylbut-2-en-1-yl)oxy)silanol

Compound 1q: Previously characterized in Org. Lett. 2020, 22, 8665-8669.

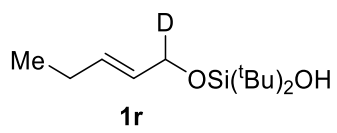

(E)-di-tert-butyl((pent-2-en-1-yl-1-d)oxy)silanol

Compound 1r: Previously characterized in Org. Lett. 2020, 22, 8665-8669.

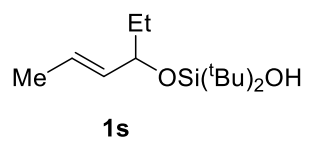

(E)-di-tert-butyl(hex-4-en-3-yloxy)silanol

Compound 1s: Previously characterized in Org. Lett. 2020, 22, 8665-8669.

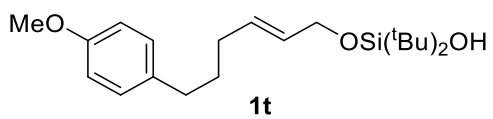

(E)-di-tert-butyl((6-(4-methoxyphenyl)hex-2-en-1-yl)oxy)silanol

Compound 1t: Purified using a gradient of 0 to $2 \%$ acetone/DCM on silica gel; (colorless oil, $339 \mathrm{mg}$, $46 \%$ yield); ${ }^{1} \mathrm{H}$ NMR (400 MHz, $\left.\mathrm{CDCl}_{3}\right) \delta 7.13(\mathrm{dd}, J=8.7,2.2 \mathrm{~Hz}, 2 \mathrm{H}), 6.93-6.79(\mathrm{~m}, 2 \mathrm{H}), 5.80$ - $5.67(\mathrm{~m}, 1 \mathrm{H}), 5.62$ (ddddd, $J=15.3,5.2,4.0,2.6,1.3 \mathrm{~Hz}, 1 \mathrm{H}), 4.34(\mathrm{ddt}, J=5.3,4.0,1.2 \mathrm{~Hz}, 2 \mathrm{H}), 3.82(\mathrm{~s}, 3 \mathrm{H}), 2.60(\mathrm{td}, J=7.8,2.3 \mathrm{~Hz}, 2 \mathrm{H})$, $2.16-2.05(\mathrm{~m}, 2 \mathrm{H}), 1.77-1.63(\mathrm{~m}, 2 \mathrm{H}), 1.10-0.98(\mathrm{~m}, 18 \mathrm{H}) .{ }^{13} \mathrm{C}$ NMR $\left(100 \mathrm{MHz}, \mathrm{CDCl}_{3}\right) \delta 157.7,134.5,130.7$, 129.7, 129.3, 113.7, 64.1, 55.2, 34.4, 31.6, 31.2, 27.4, 20.5. IR 3522, 2951, 2854, 1513, 1473, 1244, $1044 \mathrm{~cm}^{-1}$.; HRMS (APCI) m/z: [M+] calculated for $\mathrm{C}_{21} \mathrm{H}_{36} \mathrm{O}_{3} \mathrm{Si} 364.2434$, Found 364.2422.

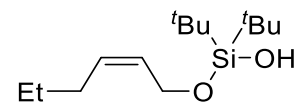

$1 \mathrm{u}$

(Z)-di-tert-butyl(hex-2-en-1-yloxy)silanol

Compound 1u: Purified using a gradient of 0 to $0.2 \%$ acetone/DCM on silica gel; (colorless oil, $252 \mathrm{mg}$, $48 \%$ yield); ${ }^{1} \mathrm{H}$ NMR $\left(400 \mathrm{MHz}, \mathrm{CDCl}_{3}\right) \delta 5.59(\mathrm{dtt}, J=11.0,6.1,1.5 \mathrm{~Hz}, 1 \mathrm{H}), 5.51-5.40(\mathrm{~m}, 1 \mathrm{H}), 4.47-4.36(\mathrm{~m}, 2 \mathrm{H}), 2.05$ (qd, $J=7.4,1.4 \mathrm{~Hz}, 2 \mathrm{H}), 1.41(\mathrm{~h}, J=7.4 \mathrm{~Hz}, 2 \mathrm{H}), 1.05(\mathrm{~s}, 18 \mathrm{H}), 0.92(\mathrm{t}, J=7.4 \mathrm{~Hz}, 3 \mathrm{H}) .{ }^{13} \mathrm{C}\{1 \mathrm{H}\} \mathrm{NMR}(100 \mathrm{MHz}$, 
$\left.\mathrm{CDCl}_{3}\right) \delta 130.7,129.7,59.6,29.5,27.4,22.7,20.4,13.7$. IR $3494,2951,2854,1467,1090,833 \mathrm{~cm}^{-1}$.; HRMS (APCI) $\mathrm{m} / \mathrm{z}:[\mathrm{M}-\mathrm{H}]$ calculated for $\mathrm{C}_{14} \mathrm{H}_{29} \mathrm{O}_{2} \mathrm{Si} 257.1937$, found 257.1935 .

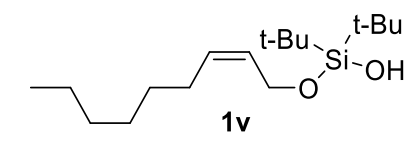

(Z)-di-tert-butyl(non-2-en-1-yloxy)silanol

Compound 1v: Purified using a gradient of 0 to $0.2 \%$ acetone/DCM on silica gel; (colorless oil, $324 \mathrm{mg}$, 54\% yield); ${ }^{1} \mathrm{H}$ NMR (400 MHz, $\left.\mathrm{CDCl}_{3}\right) \delta 5.58(\mathrm{dtt}, J=11.0,6.0,1.5 \mathrm{~Hz}, 1 \mathrm{H}), 5.52-5.38(\mathrm{~m}, 1 \mathrm{H}), 4.42(\mathrm{ddt}, J=6.1,1.6,0.8$ $\mathrm{Hz}, 2 \mathrm{H}), 2.12-2.02(\mathrm{~m}, 2 \mathrm{H}), 1.44-1.25(\mathrm{~m}, 8 \mathrm{H}), 1.05(\mathrm{~s}, 18 \mathrm{H}), 0.95-0.85(\mathrm{~m}, 3 \mathrm{H}) .{ }^{13} \mathrm{C}\{1 \mathrm{H}\} \mathrm{NMR}(100 \mathrm{MHz}$, $\left.\mathrm{CDCl}_{3}\right) \delta 131.0,129.5,59.6,31.7,29.5,28.9,27.5,27.4,22.6,20.4,14.0$. IR 3500, 2946, 2866, 1473, 1096, $822 \mathrm{~cm}^{-}$

${ }^{1}$;; HRMS (APCI) m/z: [M+ $\left.\mathrm{H}^{+}\right]$calculated for $\mathrm{C}_{17} \mathrm{H}_{37} \mathrm{O}_{2} \mathrm{Si} 301.2557$, found 301.2549.

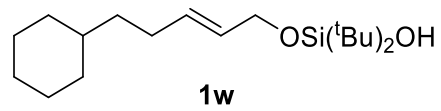

(E)-di-tert-butyl((5-cyclohexylpent-2-en-1-yl)oxy)silanol

Compound 1w: Purified using a gradient of 0.5 to 5\% diethyl ether/hexane on silica gel; (colorless oil, $592 \mathrm{mg}, 50 \%$ yield). ${ }^{1} \mathrm{H}$ NMR (400 MHz, $\left.\mathrm{CDCl}_{3}\right) \delta 5.73-5.61(\mathrm{~m}, 1 \mathrm{H}), 5.61-5.50(\mathrm{~m}, 1 \mathrm{H}), 4.33-4.23(\mathrm{~m}, 2 \mathrm{H}), 2.09-1.98(\mathrm{~m}$, $2 \mathrm{H}), 1.83(\mathrm{~s}, 1 \mathrm{H}), 1.73-1.60(\mathrm{~m}, 5 \mathrm{H}), 1.27-1.13(\mathrm{~m}, 6 \mathrm{H}), 1.02(\mathrm{~s}, 18 \mathrm{H}), 0.89-0.84(\mathrm{~m}, 1 \mathrm{H}),{ }^{13} \mathrm{C}\left\{{ }^{1} \mathrm{H}\right\} \mathrm{NMR}(100$ $\left.\mathrm{MHz}, \mathrm{CDCl}_{3}\right) \delta 131.8,129.1,64.3,37.2,37.0,33.4,29.6,27.5,26.8,26.5,20.6$. IR 3494, 2923, 2854, 1473, 1450, 1381, 1364, 1113, 1056, 970, 827, $645 \mathrm{~cm}^{-1}$.; HRMS (APCI) m/z: $\left[\mathrm{M}+\mathrm{H}^{+}\right]$calculated for $\mathrm{C}_{19} \mathrm{H}_{39} \mathrm{O}_{2} \mathrm{Si} 327.2719$, found 327.2719 .

\section{General Procedure for Tethered Silanoxyselenylation}

0.1 mmol scale: An oven dried tube equipped with a magnetic stir bar was charged with allylic silanol starting material (0.1 mmol) and $0.5 \mathrm{~mL} \mathrm{CH}_{2} \mathrm{Cl}_{2}$. Next, 10-camphorsulfonic acid (0.1 mmol, $23 \mathrm{mg}, 1.0$ equiv.), $N$ (Phenylseleno)phthalimide ( $0.15 \mathrm{mmol}, 45 \mathrm{mg}, 1.5$ equiv.), and $0.5 \mathrm{~mL} \mathrm{CH}_{2} \mathrm{Cl}_{2}$ were added to the above solution sequentially (Final reaction concentration: $0.1 \mathrm{M}$ ). The mixture was stirred at room temperature and monitored by thin layer chromatography (TLC). After $2.0 \mathrm{~h}$, the reaction mixture was diluted with $5 \mathrm{~mL} \mathrm{CH}_{2} \mathrm{Cl}_{2}$, transferred to a separatory funnel, and washed with $0.5 \mathrm{M}$ aqueous $\mathrm{NaOH}$ solution. The organic layer was separated, dried over $\mathrm{MgSO}_{4}$, concentrated in vacuo.

$1 \mathrm{mmol}$ scale: An oven dried tube equipped with a magnetic stir bar was charged with allylic silanol starting material 1a (1 mmol, $258 \mathrm{mg}, 1$ equiv.) and $5.0 \mathrm{~mL}$ of $\mathrm{CH}_{2} \mathrm{Cl}_{2}$. 10-camphorsulfonic acid (1 mmol, $232 \mathrm{mg}, 1.0$ equiv.) and $\mathrm{N}$ (Phenylseleno)phthalimide (1.5 mmol, $453 \mathrm{mg}, 1.5$ equiv.) were added sequentially. An additional $5.0 \mathrm{~mL} \mathrm{CH}_{2} \mathrm{Cl}_{2}$ 
were added ( $0.1 \mathrm{M}$ final concentration) and the heterogenous mixture was stirred for $3 \mathrm{~h}$. Next, the reaction mixture was diluted with $25 \mathrm{~mL} \mathrm{CH}_{2} \mathrm{Cl}_{2}$, transferred to a separatory funnel, and washed with $0.5 \mathrm{M}$ aqueous $\mathrm{NaOH}$ solution. The organic layer was separated, dried over $\mathrm{MgSO}_{4}$, concentrated in vacuo.

\section{Tethered Silanoxyselenylation Product Characterization (Scheme 2 Compounds)}

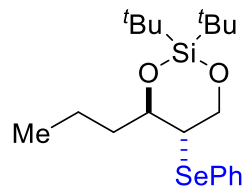

$2 \mathbf{a}$

2,2-di-tert-butyl-5-(phenylselanyl)-4-propyl-1,3,2-dioxasilinane

Compound 2a: Synthesized using the general procedure; single diastereomer; (pale yellow oil, 78\% NMR yield); ${ }^{1} \mathrm{H}$ NMR (400 MHz, $\left.\mathrm{CDCl}_{3}\right) \delta 7.49-7.45(\mathrm{~m}, 2 \mathrm{H}), 7.23-7.17(\mathrm{~m}, 3 \mathrm{H}), 4.06(\mathrm{dd}, J=11.3,4.6 \mathrm{~Hz}, 1 \mathrm{H}), 3.97(\mathrm{t}, J=11.5$ $\mathrm{Hz}, 1 \mathrm{H}), 3.90(\mathrm{ddd}, J=10.7,8.4,2.4 \mathrm{~Hz}, 1 \mathrm{H}), 3.12(\mathrm{ddd}, J=11.6,10.7,4.6 \mathrm{~Hz}, 1 \mathrm{H}), 2.02-1.93(\mathrm{~m}, 1 \mathrm{H}), 1.53-1.33$ $(\mathrm{m}, 3 \mathrm{H}), 0.92(\mathrm{~s}, 9 \mathrm{H}), 0.90(\mathrm{~s}, 9 \mathrm{H}), 0.86(\mathrm{t}, J=7.2 \mathrm{~Hz}, 3 \mathrm{H}) .{ }^{13} \mathrm{C}\left\{{ }^{1} \mathrm{H}\right\} \mathrm{NMR}\left(100 \mathrm{MHz}, \mathrm{CDCl}_{3}\right) \delta 135.2,129.3,128.2$, 127.2, 76.9, 69.1, 47.4, 39.0, 27.6, 27.1, 22.8, 19.9, 18.1, 14.0. IR 2957, 2934, 2860, 1473, 1141, 1073, 1022, 827, 787, 736, $650 \mathrm{~cm}^{-1}$; HRMS (APCI) m/z: $\left[\mathrm{M}+\mathrm{H}^{+}\right]$calculated for $\mathrm{C}_{20} \mathrm{H}_{35} \mathrm{O}_{2} \mathrm{SeSi} 415.1566$, found 415.1563.

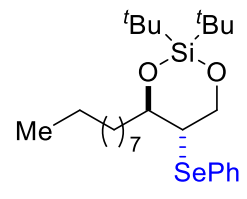

2b

2,2-di-tert-butyl-5-(phenylselanyl)-4-propyl-1,3,2-dioxasilinane

Compound 2b: Synthesized using the general procedure; single diastereomer; (pale yellow oil, 81\% NMR yield); ${ }^{1} \mathrm{H}$ $\operatorname{NMR}\left(400 \mathrm{MHz} \mathrm{CDCl}_{3}\right) \delta 7.49-7.43(\mathrm{~m}, 2 \mathrm{H}), 7.21-7.17(\mathrm{~m}, 3 \mathrm{H}), 4.06(\mathrm{dd}, J=11.3,4.6 \mathrm{~Hz}, 1 \mathrm{H}), 3.96(\mathrm{t}, J=11.5$ $\mathrm{Hz}, 1 \mathrm{H}), 3.88$ (ddd, $J=10.7,8.4,2.4 \mathrm{~Hz}, 1 \mathrm{H}), 3.12$ (ddd, $J=11.6,10.8,4.6 \mathrm{~Hz}, 1 \mathrm{H}), 2.08-1.91$ (m, $1 \mathrm{H}), 1.49-1.42$ $(\mathrm{m}, 1 \mathrm{H}), 1.20(\mathrm{~s}, 14 \mathrm{H}), 0.92(\mathrm{~s}, 9 \mathrm{H}), 0.90(\mathrm{~s}, 9 \mathrm{H}), 0.81(\mathrm{t}, J=6.8 \mathrm{~Hz}, 3 \mathrm{H}) .{ }^{13} \mathrm{C}\left\{{ }^{1} \mathrm{H}\right\} \mathrm{NMR}\left(100 \mathrm{MHz}, \mathrm{CDCl}_{3}\right) \delta 135.2$, 129.3, 128.2, 127.3, 77.3, 69.0, 47.5, 36.8, 32.1, 29.8, 29.7, 29.5, 27.6, 27.2, 24.8, 22.9, 22.8, 19.9, 14.3. IR 2929, 2854, 1575, 1473, 1136, 1101, 1044, 827, 787, 736, 690, $650 \mathrm{~cm}^{-1}$; HRMS (APCI) m/z: [M + $\left.\mathrm{H}^{+}\right]$calculated for $\mathrm{C}_{26} \mathrm{H}_{47} \mathrm{O}_{2} \mathrm{SeSi}$ 499.2511, found 499.2521. 


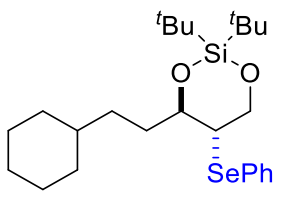

2c

2,2-di-tert-butyl-4-(2-cyclohexylethyl)-5-(phenylselanyl)-1,3,2-dioxasilinane

Compound 2c: Synthesized using the general procedure; single diastereomer; (pale yellow oil, 78\% NMR yield); ${ }^{1} \mathrm{H}$ NMR (400 MHz, $\left.\mathrm{CDCl}_{3}\right) \delta 7.49-7.44(\mathrm{~m}, 2 \mathrm{H}), 7.21-7.17$ (m, 3H), 4.05 (dd, $\left.J=11.3,4.6 \mathrm{~Hz}, 1 \mathrm{H}\right), 3.97$ (t, $J=11.5$ $\mathrm{Hz}, 1 \mathrm{H}), 3.85$ (ddd, $J=10.8,8.6,2.3 \mathrm{~Hz}, 1 \mathrm{H}), 3.12(\mathrm{ddd}, J=11.6,10.8,4.6 \mathrm{~Hz}, 1 \mathrm{H}), 2.08-1.99(\mathrm{~m}, 1 \mathrm{H}), 1.69-1.54$ $(\mathrm{m}, 6 \mathrm{H}), 1.50-1.42(\mathrm{~m}, 1 \mathrm{H}), 1.27-1.03(\mathrm{~m}, 7 \mathrm{H}), 0.92(\mathrm{~s}, 9 \mathrm{H}), 0.90(\mathrm{~s}, 9 \mathrm{H}) .{ }^{13} \mathrm{C}\left\{{ }^{1} \mathrm{H}\right\} \mathrm{NMR}\left(100 \mathrm{MHz}, \mathrm{CDCl}_{3}\right) \delta$ 135.3, 129.3, 128.2, 127.3, 77.6, 69.1, 47.5, 37.5, 34.2, 33.9, 33.4, 32.6, 27.6, 27.2, 26.9, 26.6, 26.5, 22.89, 19.9. IR 2923, 2854, 1473, 1364, 1113, 1033, 964, 827, 782, 736, 690, $650 \mathrm{~cm}^{-1}$; HRMS (APCI) m/z: [M + $\left.\mathrm{H}^{+}\right]$calculated for $\mathrm{C}_{25} \mathrm{H}_{43} \mathrm{O}_{2} \mathrm{SeSi}$ 483.2198, found 483.2203.

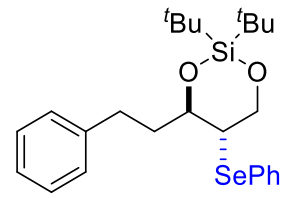

2,2-di-tert-butyl-4-phenethyl-5-(phenylselanyl)-1,3,2-dioxasilinane

Compound 2d: Synthesized using the general procedure; single diastereomer; (pale yellow oil, 60\% NMR yield); ${ }^{1} \mathrm{H}$ NMR (400 MHz, $\left.\mathrm{CDCl}_{3}\right) \delta 7.38-7.35(\mathrm{~m}, 1 \mathrm{H}), 7.22-7.13(\mathrm{~m}, 9 \mathrm{H}), 4.08(\mathrm{dd}, J=11.3,4.4 \mathrm{~Hz}, 1 \mathrm{H}), 3.90(\mathrm{t}, J=11.6$ $\mathrm{Hz}, 1 \mathrm{H}), 3.86-3.80(\mathrm{~m}, 1 \mathrm{H}), 3.09$ (ddd, $J=11.8,10.8,4.5 \mathrm{~Hz}, 1 \mathrm{H}), 2.84-2.76(\mathrm{~m}, 1 \mathrm{H}), 2.72-2.63(\mathrm{~m}, 1 \mathrm{H}), 2.47-$ $2.41(\mathrm{~m}, 1 \mathrm{H}), 1.82-1.72(\mathrm{~m}, 1 \mathrm{H}), 0.93(\mathrm{~s}, 9 \mathrm{H}), 0.91(\mathrm{~s}, 9 \mathrm{H}) .{ }^{13} \mathrm{C}\left\{{ }^{1} \mathrm{H}\right\} \mathrm{NMR}\left(100 \mathrm{MHz}, \mathrm{CDCl}_{3}\right) \delta 135.4,129.3,129.2$, 128.8, 128.4, 128.2, 127.8, 125.8, 76.0, 68.9, 47.1, 38.7, 31.1, 27.5, 27.2, 22.8, 19.9. IR 2929, 2860, 1473, 1119, 1044, 976, 827, 787, 736, 696, $650 \mathrm{~cm}^{-1}$; HRMS (APCI) m/z: $\left[\mathrm{M}+\mathrm{H}^{+}\right]$calculated for $\mathrm{C}_{25} \mathrm{H}_{37} \mathrm{O}_{2} \mathrm{SeSi}$ 477.1728, found 477.1725 .

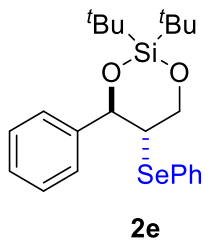

2,2-di-tert-butyl-4-phenyl-5-(phenylselanyl)-1,3,2-dioxasilinane

Compound 2e: Synthesized using the general procedure; single diastereomer; (pale yellow oil, 85\% NMR yield); ${ }^{1} \mathrm{H}$ NMR (400 MHz, $\left.\mathrm{CDCl}_{3}\right) \delta 7.34-7.31(\mathrm{~m}, 2 \mathrm{H}), 7.28-7.23(\mathrm{~m}, 3 \mathrm{H}), 7.14-7.09(\mathrm{~m}, 3 \mathrm{H}), 7.08-7.03(\mathrm{~m}, 2 \mathrm{H}), 4.93$ $(\mathrm{d}, J=10.7 \mathrm{~Hz}, 1 \mathrm{H}), 4.22-4.07(\mathrm{~m}, 2 \mathrm{H}), 3.45-3.37(\mathrm{~m}, 1 \mathrm{H}), 1.00(\mathrm{~s}, 9 \mathrm{H}), 0.98(\mathrm{~s}, 9 \mathrm{H}) .{ }^{13} \mathrm{C}\left\{{ }^{1} \mathrm{H}\right\} \mathrm{NMR}(100 \mathrm{MHz}$, $\left.\mathrm{CDCl}_{3}\right) \delta 135.2,129.3,129.0,128.3,128.2,128.0,127.8,127.4,80.9,69.5,49.2,27.7,27.3,23.0,20.2$. IR 2934, 
2860, 1473, 1273, 1261, 1101, 1033, 827, 747, 696, $650 \mathrm{~cm}^{-1}$; HRMS (APCI) m/z: [M + $\left.\mathrm{H}^{+}\right]$calculated for $\mathrm{C}_{23} \mathrm{H}_{33} \mathrm{O}_{2} \mathrm{SeSi} 449.1415$, found 449.1413 .

\section{General Procedure for Hydroxyselenylation}

An oven dried tube equipped with a magnetic stir bar was charged with allylic silanol $(0.1 \mathrm{mmol})$ and $0.5 \mathrm{~mL} \mathrm{CH}_{2} \mathrm{Cl}_{2}$. Next, sodium bicarbonate $(0.1 \mathrm{mmol}, 8 \mathrm{mg}, 1.0$ equiv. $), N$-(phenylseleno)phthalimide $(0.15 \mathrm{mmol}, 45 \mathrm{mg}, 1.5 \mathrm{equiv}$.), and $0.5 \mathrm{~mL} \mathrm{CH} \mathrm{Cl}_{2}$ were added sequentially (Final reaction concentration: $0.1 \mathrm{M}$ ). The mixture was stirred at room temperature and monitored by thin layer chromatography (TLC). After 6 h, the reaction mixture was diluted with 5 $\mathrm{mL}$ of $\mathrm{CH}_{2} \mathrm{Cl}_{2}$, transferred to a separatory funnel, and washed with water. The organic layer was separated, dried over $\mathrm{MgSO}_{4}$, concentrated in vacuo, and purified by chromatography on Florisil (specific conditions are associated with each product).

\section{Hydroxyselenylation Product Characterization (Scheme 4 Compounds)}

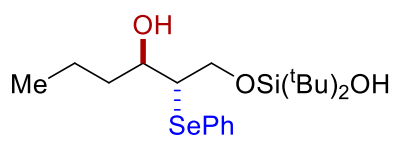

$4 a$

di-tert-butyl((3-hydroxy-2-(phenylselanyl)hexyl)oxy)silanol

Compound 4a: Synthesized using the general procedure; Purified using a gradient of 0 to $30 \%$ EtOAc/hexanes on Florisil; single diastereomer; (colorless oil, $30 \mathrm{mg}, 73 \%$ yield); ${ }^{1} \mathrm{H} \mathrm{NMR}\left(400 \mathrm{MHz}, \mathrm{CDCl}_{3}\right) \delta 7.63-7.51$ (m, $\left.2 \mathrm{H}\right)$, $7.33-7.17(\mathrm{~m}, 3 \mathrm{H}), 4.36(\mathrm{dd}, J=11.1,3.7 \mathrm{~Hz}, 1 \mathrm{H}), 4.11(\mathrm{dd}, J=11.1,6.2 \mathrm{~Hz}, 1 \mathrm{H}), 3.93(\mathrm{ddd}, J=9.0,5.8,3.4 \mathrm{~Hz}$, $1 \mathrm{H}), 3.29(\mathrm{td}, J=6.0,3.7 \mathrm{~Hz}, 1 \mathrm{H}), 1.76-1.64(\mathrm{~m}, 1 \mathrm{H}), 1.53(\mathrm{dddt}, J=17.3,8.9,5.5,4.3 \mathrm{~Hz}, 2 \mathrm{H}), 1.45-1.34(\mathrm{~m}$, $1 \mathrm{H}), 1.03(\mathrm{~s}, 9 \mathrm{H}), 1.01(\mathrm{~s}, 9 \mathrm{H}), 0.91(\mathrm{t}, J=7.2 \mathrm{~Hz}, 3 \mathrm{H}) .{ }^{13} \mathrm{C}\left\{{ }^{1} \mathrm{H}\right\} \mathrm{NMR}\left(100 \mathrm{MHz}, \mathrm{CDCl}_{3}\right) \delta 134.3,129.2,129.1$, 127.6, 72.7, 64.7, 54.4, 37.1, 27.5, 27.4, 20.6, 20.4, 19.0, 13.9. IR 3374, 2957, 2854, 1473, $1096 \mathrm{~cm}^{-1}$.; HRMS (APCI) $\mathrm{m} / \mathrm{z}$ : $\left[\mathrm{M}-\mathrm{H}_{2} \mathrm{O}+\mathrm{H}^{+}\right]$Calculated for $\mathrm{C}_{20} \mathrm{H}_{35} \mathrm{O}_{2} \mathrm{SeSi} 415.1566$, found 415.1587 .

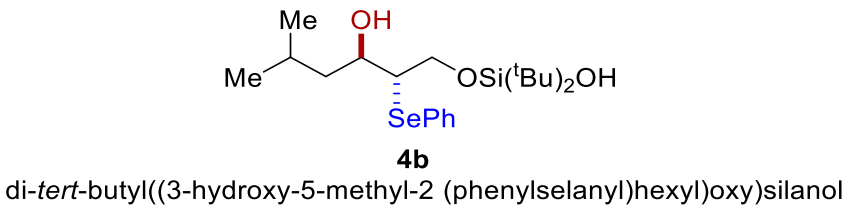

Compound 4b: Synthesized using the general procedure; Purified using a gradient of 0 to $30 \%$ EtOAc/hexanes on Florisil; single diastereomer; (colorless oil, $33 \mathrm{mg}, 78 \%$ yield); ${ }^{1} \mathrm{H}$ NMR (400 MHz, $\left.\mathrm{CDCl}_{3}\right) \delta 7.66-7.55(\mathrm{~m}, 2 \mathrm{H})$, $7.36-7.23(\mathrm{~m}, 3 \mathrm{H}), 4.38(\mathrm{dd}, J=11.1,3.8 \mathrm{~Hz}, 1 \mathrm{H}), 4.18-4.08(\mathrm{~m}, 1 \mathrm{H}), 4.08-4.00(\mathrm{~m}, 1 \mathrm{H}), 3.31(\mathrm{td}, J=6.0,3.8$ $\mathrm{Hz}, 1 \mathrm{H}), 1.94-1.81(\mathrm{~m}, 1 \mathrm{H}), 1.58-1.43(\mathrm{~m}, 2 \mathrm{H}), 1.06(\mathrm{~s}, 9 \mathrm{H}), 1.04(\mathrm{~s}, 9 \mathrm{H}), 0.93(\mathrm{~m}, 6 \mathrm{H}) .{ }^{13} \mathrm{C}\left\{{ }^{1} \mathrm{H}\right\} \mathrm{NMR}(100 \mathrm{MHz}$, $\left.\mathrm{CDCl}_{3}\right) \delta 134.3,129.3,129.1,127.6,70.8,64.5,55.2,44.0,27.5,27.4,24.7,23.6,21.6,20.6,20.4$. IR 3385, 2957, 
2860, 2358, 1473, $1096 \mathrm{~cm}^{-1}$.; HRMS (APCI) m/z: [M- $\left.\mathrm{H}_{2} \mathrm{O}+\mathrm{H}^{+}\right]$calculated for $\mathrm{C}_{21} \mathrm{H}_{37} \mathrm{O}_{2} \mathrm{SeSi}$ 429.1723, found 429.1744.

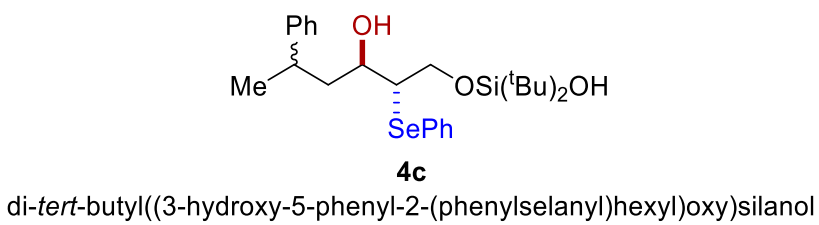

Compound 4c: Synthesized using the general procedure; Purified using a gradient of 0 to $30 \% \mathrm{EtOAc/hexanes}$ on Florisil; 1:1 mixture of diastereomers; (colorless oil, $30 \mathrm{mg}, 61 \%$ yield); ${ }^{1} \mathrm{H}$ NMR (400 MHz, $\mathrm{CDCl}_{3}$ ) $\delta 7.57-7.45$ (m, 4H), $7.32-7.15(\mathrm{~m}, 16 \mathrm{H}), 4.38(\mathrm{dd}, J=11.1,3.7 \mathrm{~Hz}, 1 \mathrm{H}), 4.32(\mathrm{dd}, J=11.1,3.6 \mathrm{~Hz}, 1 \mathrm{H}), 4.16-4.10(\mathrm{~m}, 1 \mathrm{H})$, $4.07(\mathrm{td}, J=5.8,2.9 \mathrm{~Hz}, 1 \mathrm{H}), 4.01(\mathrm{dd}, J=11.1,6.0 \mathrm{~Hz}, 1 \mathrm{H}), 3.62(\mathrm{ddd}, J=10.4,6.3,2.4 \mathrm{~Hz}, 1 \mathrm{H}), 3.30(\mathrm{td}, J=5.9$, $3.7 \mathrm{~Hz}, 1 \mathrm{H}), 3.17(\mathrm{td}, J=6.1,3.6 \mathrm{~Hz}, 1 \mathrm{H}), 3.12-2.98(\mathrm{~m}, 2 \mathrm{H}), 2.12-2.02(\mathrm{~m}, 1 \mathrm{H}), 1.98(\mathrm{ddd}, J=13.9,9.3,3.5 \mathrm{~Hz}$, 1H), $1.86-1.80(\mathrm{~m}, 1 \mathrm{H}), 1.75$ (ddd, $J=14.1,10.3,4.0 \mathrm{~Hz}, 1 \mathrm{H}), 1.32-1.23(\mathrm{~m}, 6 \mathrm{H}), 1.05$ (s, 9H), 1.03 (s, 9H), 0.97 (s, 9H), 0.93 (s, 9H). ${ }^{13} \mathrm{C}\left\{{ }^{1} \mathrm{H}\right\}$ NMR (100 MHz, $\left.\mathrm{CDCl}_{3}\right) \delta 147.7,146.4,134.4,134.3,129.4,129.3,129.2,128.7$, 128.6, 127.8, 127.7, 127.4, 127.0, 126.2, 126.1, 71.0, 70.6, 64.7, 64.6, 54.9, 54.8, 43.6, 43.5, 36.6, 36.2, 27.7, 27.6, 27.58, 27.51, 23.4, 21.2, 20.8, 20.7, 20.6, 20.5. IR 3391, 2940, 2860, 1473, 1261, $1016 \mathrm{~cm}^{-1}$; HRMS (APCI) m/z: $\left[\mathrm{M}-\mathrm{H}_{2} \mathrm{O}+\mathrm{H}^{+}\right]$calculated for $\mathrm{C}_{26} \mathrm{H}_{39} \mathrm{O}_{2} \mathrm{SeSi} 491.1879$, found 491.1879 .

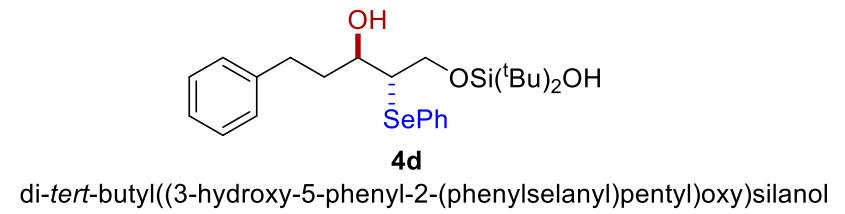

Compound 4d: Synthesized using the general procedure; Purified using a gradient of 0 to $30 \%$ EtOAc/hexanes on Florisil; single diastereomer; (colorless oil, $32 \mathrm{mg}, 69 \%$ yield); $\left.{ }^{1} \mathrm{H} \mathrm{NMR} \mathrm{(400} \mathrm{MHz,} \mathrm{CDCl}_{3}\right) \delta 7.62-7.52(\mathrm{~m}, 2 \mathrm{H})$, $7.34-7.25$ (m, 5H), 7.20 (ddt, $J=6.9,5.8,1.5 \mathrm{~Hz}, 3 \mathrm{H}), 4.39$ (dd, $J=11.1,3.7 \mathrm{~Hz}, 1 \mathrm{H}), 4.21-4.06(\mathrm{~m}, 1 \mathrm{H}), 3.97$ (ddd, $J=9.2,6.2,3.0 \mathrm{~Hz}, 1 \mathrm{H}), 3.32(\mathrm{td}, J=6.2,3.7 \mathrm{~Hz}, 1 \mathrm{H}), 2.90(\mathrm{ddd}, J=14.3,9.8,5.0 \mathrm{~Hz}, 1 \mathrm{H}), 2.80-2.65(\mathrm{~m}$, 1H), $2.20-2.02(\mathrm{~m}, 1 \mathrm{H}), 1.86(\mathrm{dtd}, J=14.2,9.4,5.1 \mathrm{~Hz}, 1 \mathrm{H}), 1.04(\mathrm{~s}, 9 \mathrm{H}), 1.02(\mathrm{~s}, 9 \mathrm{H}) .{ }^{13} \mathrm{C}\left\{{ }^{1} \mathrm{H}\right\} \mathrm{NMR}(100 \mathrm{MHz}$, $\left.\mathrm{CDCl}_{3}\right) \delta 141.9,134.4,129.2,129.1,128.5,128.3,127.7,125.8,72.2,64.9,54.1,36.7,32.0,27.5,27.4,20.6,20.4$. IR 3397, 2946, 2854, 1473, 1022, $833 \mathrm{~cm}^{-1}$.; HRMS (APCI) m/z: [M- $\left.\mathrm{H}_{2} \mathrm{O}+\mathrm{H}^{+}\right]$calculated for $\mathrm{C}_{25} \mathrm{H}_{37} \mathrm{O}_{2} \mathrm{SeSi} 477.1723$, found 477.1741 . 


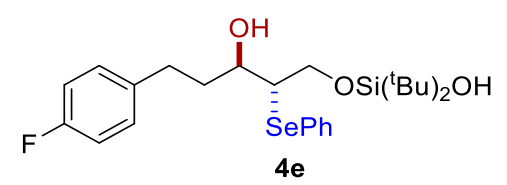

di-tert-butyl((5-(4-fluorophenyl)-3-hydroxy-2-(phenylselanyl)pentyl)oxy)silanol

Compound 4e: Synthesized using the general procedure; Purified using a gradient of 0 to $30 \%$ EtOAc/hexanes on Florisil; single diastereomer; (colorless oil, $33 \mathrm{mg}, 67 \%$ yield); ${ }^{1} \mathrm{H} \mathrm{NMR}\left(400 \mathrm{MHz}, \mathrm{CDCl}_{3}\right) \delta 7.62-7.50(\mathrm{~m}, 2 \mathrm{H})$, $7.35-7.22(\mathrm{~m}, 3 \mathrm{H}), 7.14$ (ddd, $J=8.7,5.5,2.6 \mathrm{~Hz}, 2 \mathrm{H}), 7.03-6.89(\mathrm{~m}, 2 \mathrm{H}), 4.40(\mathrm{dd}, J=11.1,3.7 \mathrm{~Hz}, 1 \mathrm{H}), 4.17-$ $4.06(\mathrm{~m}, 1 \mathrm{H}), 3.93(\mathrm{ddd}, J=9.3,6.3,2.9 \mathrm{~Hz}, 1 \mathrm{H}), 3.30(\mathrm{td}, J=6.3,3.7 \mathrm{~Hz}, 1 \mathrm{H}), 2.86(\mathrm{ddd}, J=14.2,9.5,5.0 \mathrm{~Hz}, 1 \mathrm{H})$, $2.70(\mathrm{ddd}, J=14.6,9.5,7.6 \mathrm{~Hz}, 1 \mathrm{H}), 2.12-2.01(\mathrm{~m}, 1 \mathrm{H}), 1.83(\mathrm{dtd}, J=14.1,9.3,5.0 \mathrm{~Hz}, 1 \mathrm{H}), 1.04(\mathrm{~s}, 9 \mathrm{H}), 1.02(\mathrm{~s}$, 9H). ${ }^{13} \mathrm{C}\left\{{ }^{1} \mathrm{H}\right\} \operatorname{NMR}\left(125 \mathrm{MHz}, \mathrm{CDCl}_{3}\right) \delta 161.4(\mathrm{~d}, J=243.4 \mathrm{~Hz}), 137.6(\mathrm{~d}, J=2.8 \mathrm{~Hz}), 134.5,130.0(\mathrm{~d}, J=7.0 \mathrm{~Hz})$, 129.3, 129.1, 127.9, 115.2 (d, $J=20.9$ Hz), 72.0, 65.0, 54.1, 36.8, 31.2, 27.6, 27.5, 20.7, 20.5. IR 3397, 2940, 2854, 1507, 1473, 1221, $1107 \mathrm{~cm}^{-1}$; HRMS (APCI) m/z: [M- $\left.\mathrm{H}_{2} \mathrm{O}+\mathrm{H}^{+}\right]$calculated for $\mathrm{C}_{25} \mathrm{H}_{36} \mathrm{FO}_{2} \mathrm{SeSi}$ 495.1628, found 495.1646.

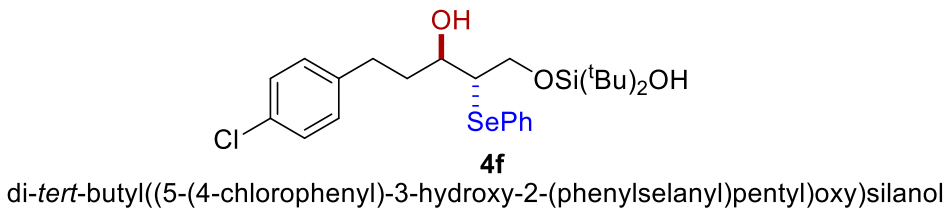

Compound 4f: Synthesized using the general procedure; Purified using a gradient of 0 to $30 \%$ EtOAc/hexanes on Florisil; single diastereomer; (colorless oil, $37 \mathrm{mg}, 70 \%$ yield); ${ }^{1} \mathrm{H} \mathrm{NMR}\left(400 \mathrm{MHz}, \mathrm{CDCl}_{3}\right) \delta 7.58-7.50(\mathrm{~m}, 2 \mathrm{H})$, $7.36-7.16$ (m, 5H), $7.13-7.06(\mathrm{~m}, 2 \mathrm{H}), 4.37$ (dd, $J=11.0,3.7 \mathrm{~Hz}, 1 \mathrm{H}), 4.18-4.03$ (m, 1H), 3.89 (ddd, $J=9.3,6.4$, $2.9 \mathrm{~Hz}, 1 \mathrm{H}), 3.26(\mathrm{td}, J=6.4,3.6 \mathrm{~Hz}, 1 \mathrm{H}), 2.82(\mathrm{ddd}, J=14.1,9.5,4.9 \mathrm{~Hz}, 1 \mathrm{H}), 2.68(\mathrm{ddd}, J=14.2,9.3,7.6 \mathrm{~Hz}, 1 \mathrm{H})$, $2.08-1.97(\mathrm{~m}, 1 \mathrm{H}), 1.80(\mathrm{dtd}, J=14.1,9.2,5.0 \mathrm{~Hz}, 1 \mathrm{H}), 1.01(\mathrm{~s}, 9 \mathrm{H}), 0.99(\mathrm{~s}, 9 \mathrm{H}) .{ }^{13} \mathrm{C}\left\{{ }^{1} \mathrm{H}\right\} \mathrm{NMR}\left(100 \mathrm{MHz}, \mathrm{CDCl}_{3}\right)$ $\delta 140.4,134.5,131.6,130.0,129.3,129.0,128.5,127.9,72.0,65.1,54.1,36.6,31.4,27.6,27.5,20.7,20.5$. IR 3391, 2940, 2860, 1473, 1090, $1016 \mathrm{~cm}^{-1}$; HRMS (APCI) m/z: [M- $\left.\mathrm{H}_{2} \mathrm{O}+\mathrm{H}^{+}\right]$calculated for $\mathrm{C}_{25} \mathrm{H}_{36} \mathrm{ClO}_{2} \mathrm{SeSi} 511.1333$, found 511.1353 .

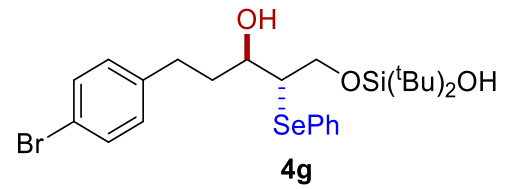

Compound 4g: Synthesized using the general procedure; Purified using a gradient of 0 to $30 \%$ EtOAc/hexanes on Florisil; single diastereomer; (colorless oil, $39 \mathrm{mg}, 71 \%$ yield); ${ }^{1} \mathrm{H} \mathrm{NMR}\left(500 \mathrm{MHz}, \mathrm{CDCl}_{3}\right) \delta 7.45-7.38(\mathrm{~m}, 2 \mathrm{H})$, $7.28-7.23(\mathrm{~m}, 2 \mathrm{H}), 7.20-7.11(\mathrm{~m}, 3 \mathrm{H}), 6.93(\mathrm{~d}, J=8.3 \mathrm{~Hz}, 2 \mathrm{H}), 4.25(\mathrm{dd}, J=11.1,3.7 \mathrm{~Hz}, 1 \mathrm{H}), 4.00-3.93(\mathrm{~m}$, 1H), 3.77 (ddd, $J=9.4,6.4,2.8 \mathrm{~Hz}, 1 \mathrm{H}), 3.14$ (td, $J=6.4,3.6 \mathrm{~Hz}, 1 \mathrm{H}), 2.69$ (ddd, $J=14.1,9.4,4.9 \mathrm{~Hz}, 1 \mathrm{H}), 2.60-$ $2.50(\mathrm{~m}, 1 \mathrm{H}), 1.96-1.87(\mathrm{~m}, 1 \mathrm{H}), 1.68(\mathrm{dtd}, J=14.0,9.2,4.9 \mathrm{~Hz}, 1 \mathrm{H}), 0.90(\mathrm{~s}, 9 \mathrm{H}), 0.88-0.84(\mathrm{~m}, 9 \mathrm{H}) .{ }^{13} \mathrm{C}\left\{{ }^{1} \mathrm{H}\right\}$ 
NMR $\left(125 \mathrm{MHz}, \mathrm{CDCl}_{3}\right) \delta 140.8,134.4,131.4,130.3,129.2,128.9,127.8,119.5,71.8,64.9,53.9,36.4,31.3,27.5$, 27.3, 20.6, 20.4. IR 3408, 2934, 2860, 1473, 1267, $1107 \mathrm{~cm}^{-1}$.; HRMS (APCI) m/z: $\left[\mathrm{M}-\mathrm{H}_{2} \mathrm{O}+\mathrm{H}^{+}\right.$] calculated for $\mathrm{C}_{25} \mathrm{H}_{36} \mathrm{BrO}_{2} \mathrm{SeSi} 555.0828$, found 555.0854.

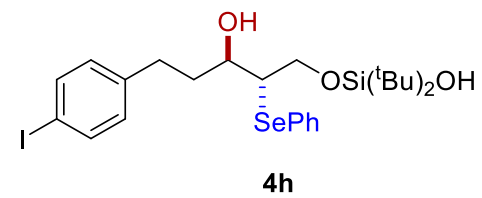

di-tert-butyl((3-hydroxy-5-(4-iodophenyl)-2-(phenylselanyl)pentyl)oxy)silanol

Compound 4h: Synthesized using the general procedure; Purified using a gradient of 0 to $30 \%$ EtOAc/hexanes on Florisil; single diastereomer; (colorless oil, $44 \mathrm{mg}, 71 \%$ yield); ${ }^{1} \mathrm{H} \mathrm{NMR}\left(400 \mathrm{MHz}, \mathrm{CDCl}_{3}\right) \delta 7.68-7.47$ (m, 4H), $7.34-7.19$ (m, 3H), $6.99-6.86(\mathrm{~m}, 2 \mathrm{H}), 4.36(\mathrm{dd}, J=11.1,3.7 \mathrm{~Hz}, 1 \mathrm{H}), 4.09$ (td, $J=11.7,6.8 \mathrm{~Hz}, 1 \mathrm{H}), 3.87$ (ddd, $J$ $=9.3,6.3,2.8 \mathrm{~Hz}, 1 \mathrm{H}), 3.25(\mathrm{td}, J=6.4,3.7 \mathrm{~Hz}, 1 \mathrm{H}), 2.79(\mathrm{ddd}, J=13.9,9.3,4.8 \mathrm{~Hz}, 1 \mathrm{H}), 2.72-2.56(\mathrm{~m}, 1 \mathrm{H}), 2.08$ $-1.97(\mathrm{~m}, 1 \mathrm{H}), 1.79(\mathrm{dtd}, J=14.1,9.2,5.0 \mathrm{~Hz}, 1 \mathrm{H}), 1.01(\mathrm{~s}, 9 \mathrm{H}), 0.98(\mathrm{~s}, 9 \mathrm{H}) .{ }^{13} \mathrm{C}\left\{{ }^{1} \mathrm{H}\right\} \mathrm{NMR}\left(100 \mathrm{MHz}, \mathrm{CDCl}_{3}\right) \delta$ 141.5, 137.3, 134.4, 130.7, 129.2, 128.9, 127.8, 90.8, 71.8, 65.0, 53.9, 36.4, 31.4, 27.5, 27.4, 20.6, 20.4. IR 3397, 2946, 2860, 1473, $1101 \mathrm{~cm}^{-1}$; HRMS (APCI) m/z: [M- $\left.\mathrm{H}_{2} \mathrm{O}+\mathrm{H}^{+}\right]$calculated for $\mathrm{C}_{25} \mathrm{H}_{36} \mathrm{IO}_{2} \mathrm{SeSi} 603.0689$, found 603.0710 .

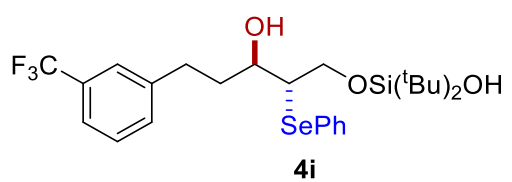

di-tert-butyl((3-hydroxy-2-(phenylselanyl)-5-(3-(trifluoromethyl)phenyl)pentyl)oxy)silanol

Compound 4i: Synthesized using the general procedure; Purified using a gradient of 0 to $30 \%$ EtOAc/hexanes on Florisil; single diastereomer; (colorless oil, $34 \mathrm{mg}, 60 \%$ yield); ${ }^{1} \mathrm{H} \mathrm{NMR}\left(400 \mathrm{MHz}, \mathrm{CDCl}_{3}\right) \delta 7.61-7.54(\mathrm{~m}, 2 \mathrm{H}$ ), $7.51-7.36$ (m, 4H), $7.34-7.24$ (m, 3H), 4.42 (dd, $J=11.1,3.6 \mathrm{~Hz}, 1 \mathrm{H}), 4.13$ (dd, $J=11.0,6.3 \mathrm{~Hz}, 1 \mathrm{H}), 3.94$ (ddd, $J=9.4,6.6,2.8 \mathrm{~Hz}, 1 \mathrm{H}), 3.30(\mathrm{td}, J=6.4,3.6 \mathrm{~Hz}, 1 \mathrm{H}), 2.93$ (ddt, $J=17.5,12.5,6.3 \mathrm{~Hz}, 1 \mathrm{H}), 2.79$ (ddd, $J=13.8,9.4$, $7.1 \mathrm{~Hz}, 1 \mathrm{H}), 2.17-2.06(\mathrm{~m}, 1 \mathrm{H}), 1.86(\mathrm{dtd}, J=14.2,9.4,4.9 \mathrm{~Hz}, 1 \mathrm{H}), 1.04(\mathrm{~s}, 9 \mathrm{H}), 1.01(\mathrm{~s}, 9 \mathrm{H}) .{ }^{13} \mathrm{C}\left\{{ }^{1} \mathrm{H}\right\} \mathrm{NMR}(100$ $\left.\mathrm{MHz}, \mathrm{CDCl}_{3}\right) \delta 142.8,134.4,131.9,130.6(\mathrm{q}, J=31.8 \mathrm{~Hz}), 129.2,128.9,128.7,127.8,125.6(\mathrm{q}, J=270 \mathrm{~Hz}), 125.3$ - 125.1 (m), 122.8 - 122.6 (m), 71.9, 65.0, 53.9, 36.4, 31.7, 27.5, 27.4, 20.6, 20.4. IR 3391, 2934, 2860, 1478, 1330, $1130 \mathrm{~cm}^{-1}$; HRMS (APCI) m/z: [M- $\left.\mathrm{H}_{2} \mathrm{O}+\mathrm{H}^{+}\right]$calculated for $\mathrm{C}_{26} \mathrm{H}_{36} \mathrm{~F}_{3} \mathrm{O}_{2} \mathrm{SeSi} 545.1602$, found 545.1625. 


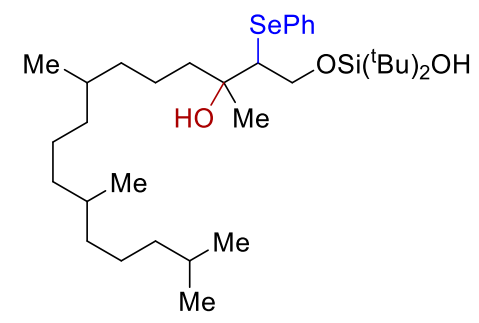

4j

di-tert-butyl((3-hydroxy-3,7,11,15-tetramethyl-2 (phenylselanyl)hexadecyl)oxy)silanol

Compound 4j: Synthesized using the general procedure; Purified using a gradient of 0 to $30 \%$ EtOAc/hexanes on Florisil; mixture of diastereomers; (colorless oil, $18 \mathrm{mg}, 30 \%$ yield); ${ }^{1} \mathrm{H}$ NMR (400 MHz, $\left.\mathrm{CDCl}_{3}\right) \delta 7.69-7.54$ (m, $2 \mathrm{H}), 7.37-7.18(\mathrm{~m}, 3 \mathrm{H}), 4.37-4.25(\mathrm{~m}, 2 \mathrm{H}), 3.36-3.27(\mathrm{~m}, 1 \mathrm{H}), 1.78-1.08(\mathrm{~m}, 24 \mathrm{H}), 1.06(\mathrm{~s}, 9 \mathrm{H}), 1.01(\mathrm{~s}, 9 \mathrm{H})$, $0.93-0.82(\mathrm{~m}, 12 \mathrm{H}) .{ }^{13} \mathrm{C}\left\{{ }^{1} \mathrm{H}\right\} \mathrm{NMR}\left(100 \mathrm{MHz}, \mathrm{CDCl}_{3}\right) \delta 134.2,129.9,129.2,127.6,75.3,65.6,57.3,41.9,39.3$, 37.6, 37.5, 37.48, 37.46, 37.3, 32.8, 32.7, 27.9, 27.4, 25.1, 24.8, 24.52, 24.51, 22.7, 22.6, 20.9, 20.5, 20.4, 19.7. IR $3408,2929,2860,1467,1376,827 \mathrm{~cm}^{-1}$.; HRMS (APCI) m/z: [M- $\left.\mathrm{H}_{2} \mathrm{O}+\mathrm{H}^{+}\right]$calculated for $\mathrm{C}_{34} \mathrm{H}_{63} \mathrm{O}_{2} \mathrm{SeSi} 611.3757$, found 611.3786 .

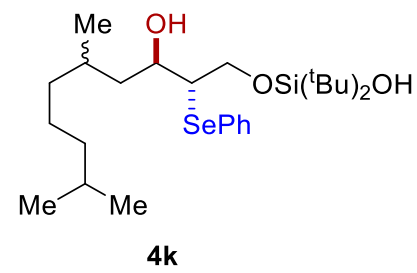

di-tert-butyl((3-hydroxy-5,9-dimethyl-2-(phenylselanyl)decyl)oxy)silanol

Compound 4k: Synthesized using the general procedure; Purified using a gradient of 0 to $30 \%$ EtOAc/hexanes on Florisil; mixture of diastereomers; (colorless oil, $33 \mathrm{mg}, 64 \%$ yield); ${ }^{1} \mathrm{H}$ NMR (400 MHz, $\left.\mathrm{CDCl}_{3}\right) \delta 7.65-7.56$ (m, 2H), $7.34-7.25(\mathrm{~m}, 3 \mathrm{H}), 4.39$ (ddd, $J=11.1,3.7,1.0 \mathrm{~Hz}, 1 \mathrm{H}), 4.17-4.01(\mathrm{~m}, 2 \mathrm{H}), 3.34-3.26(\mathrm{~m}, 1 \mathrm{H}), 1.72-1.11$ (m, 10H), $1.05(\mathrm{~m}, 18 \mathrm{H}), 0.94-0.85(\mathrm{~m}, 9 \mathrm{H}) .{ }^{13} \mathrm{C}\left\{{ }^{1} \mathrm{H}\right\} \mathrm{NMR}\left(100 \mathrm{MHz}, \mathrm{CDCl}_{3}\right) \delta 134.4,134.3,129.4,129.2,127.7$, 71.0, 70.6, 64.6, 64.4, 55.4, 55.1, 42.5, 42.3, 39.4, 39.3, 38.2, 36.3, 29.8, 29.3, 28.0, 27.9, 27.6, 27.5, 27.4, 24.7, 24.5, 22.8, 22.7, 22.6, 22.5, 20.6, 20.5, 20.4, 19.0. IR 3391, 2934, 2860, 1473, 1107, $827 \mathrm{~cm}^{-1}$.; HRMS (APCI) m/z: [M$\left.\mathrm{H}_{2} \mathrm{O}+\mathrm{H}^{+}\right]$calculated for $\mathrm{C}_{26} \mathrm{H}_{47} \mathrm{O}_{2} \mathrm{SeSi} 499.2511$, found 499.2521 .

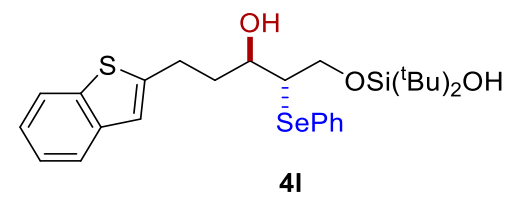

((5-(benzo[b]thiophen-2-yl)-3-hydroxy-2-(phenylselanyl)pentyl)oxy)di-tert-butylsilanol

Compound 4I: Synthesized using the general procedure; Purified using a gradient of 0 to $30 \%$ EtOAc/hexanes on Florisil; single diastereomer; (colorless oil, $36 \mathrm{mg}, 68 \%$ yield); ${ }^{1} \mathrm{H} \mathrm{NMR}\left(600 \mathrm{MHz}, \mathrm{CDCl}_{3}\right) \delta 7.78(\mathrm{~d}, J=7.9 \mathrm{~Hz}$, 
1H), $7.68(\mathrm{~d}, J=7.9 \mathrm{~Hz}, 1 \mathrm{H}), 7.58(\mathrm{dt}, J=7.0,1.5 \mathrm{~Hz}, 2 \mathrm{H}), 7.35-7.31(\mathrm{~m}, 1 \mathrm{H}), 7.29-7.21(\mathrm{~m}, 4 \mathrm{H}), 7.03(\mathrm{~s}, 1 \mathrm{H})$, $4.43(\mathrm{dd}, J=11.1,3.6 \mathrm{~Hz}, 1 \mathrm{H}), 4.15(\mathrm{dd}, J=11.3,6.4 \mathrm{~Hz}, 1 \mathrm{H}), 4.03$ (ddd, $J=9.6,6.4,2.9 \mathrm{~Hz}, 1 \mathrm{H}), 3.32$ (td, $J=6.3$, $3.6 \mathrm{~Hz}, 1 \mathrm{H}), 3.17$ (ddd, $J=14.4,9.1,5.1 \mathrm{~Hz}, 1 \mathrm{H}), 3.06(\mathrm{dt}, J=15.4,8.1 \mathrm{~Hz}, 1 \mathrm{H}), 2.25(\mathrm{dddd}, J=13.8,9.0,7.6,2.8$ $\mathrm{Hz}, 1 \mathrm{H}), 1.97$ (dtd, $J=13.9,9.1,5.0 \mathrm{~Hz}, 1 \mathrm{H}), 1.03(\mathrm{~s}, 9 \mathrm{H}), 1.01(\mathrm{~s}, 9 \mathrm{H}) .{ }^{13} \mathrm{C}\left\{{ }^{1} \mathrm{H}\right\} \mathrm{NMR}\left(100 \mathrm{MHz}, \mathrm{CDCl}_{3}\right) \delta 145.7$, 140.2, 139.4, 134.4, 129.2, 128.9, 127.7, 124.0, 123.4, 122.7, 122.1, 121.0, 71.9, 65.0, 53.9, 36.3, 27.4, 27.3, 27.0, 20.6, 20.4. IR 3402, 2934, 2860, 1473, 1107, $827 \mathrm{~cm}^{-1}$; HRMS (APCI) m/z: [M- $\mathrm{H}_{2} \mathrm{O}$ ] calculated for $\mathrm{C}_{27} \mathrm{H}_{36} \mathrm{O}_{2} \mathrm{SSeSi}$ 532.1370 , found 532.1406 .

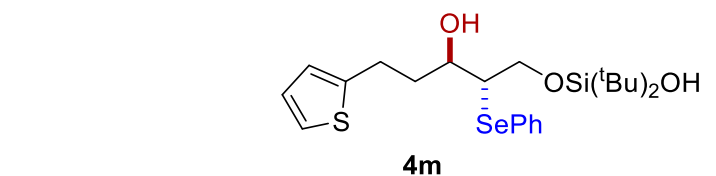

di-tert-butyl((3-hydroxy-2-(phenylselanyl)-5-(thiophen-2-yl)pentyl)oxy)silanol

Compound 4m: Synthesized using the general procedure; Purified using a gradient of 0 to $30 \% \mathrm{EtOAc/hexanes}$ on Florisil; single diastereomer; (colorless oil, $19.1 \mathrm{mg}, 38 \%$ yield); ${ }^{1} \mathrm{H}$ NMR (400 MHz, $\left.\mathrm{CDCl}_{3}\right) \delta 7.50-7.47$ (m, 2H), $7.21-7.17(\mathrm{~m}, 3 \mathrm{H}), 7.03(\mathrm{dd}, J=5.1,1.2 \mathrm{~Hz}, 1 \mathrm{H}), 6.82(\mathrm{dd}, J=5.1,3.4 \mathrm{~Hz}, 1 \mathrm{H}), 6.72-6.68(\mathrm{~m}, 1 \mathrm{H}), 4.31(\mathrm{dd}, J=$ $11.1,3.6 \mathrm{~Hz}, 1 \mathrm{H}), 4.03(\mathrm{dd}, J=11.1,6.2 \mathrm{~Hz}, 1 \mathrm{H}), 3.93-3.87(\mathrm{~m}, 1 \mathrm{H}), 3.25-3.16(\mathrm{~m}, 1 \mathrm{H}), 3.04-2.95(\mathrm{~m}, 1 \mathrm{H}), 2.91$ $-2.81(\mathrm{~m}, 1 \mathrm{H}), 2.12-2.02(\mathrm{~m}, 1 \mathrm{H}), 1.85-1.74(\mathrm{~m}, 1 \mathrm{H}), 0.94(\mathrm{~s}, 9 \mathrm{H}), 0.92(\mathrm{~s}, 9 \mathrm{H}) .{ }^{13} \mathrm{C}\left\{{ }^{1} \mathrm{H}\right\} \mathrm{NMR}\left(100 \mathrm{MHz}, \mathrm{CDCl}_{3}\right)$ $\delta 144.8,134.5,129.3,127.8,126.8,124.5,123.1,72.1,64.9,54.1,37.1,27.6,27.5,26.3,20.7,20.5$. IR 3380, 2929, 2854, 1473, 1107, 827, $690 \mathrm{~cm}^{-1}$; HRMS (APCI) m/z: [M-H] calculated for $\mathrm{C}_{23} \mathrm{H}_{35} \mathrm{O}_{3} \mathrm{SSeSi} 499.1241$, found 499.1230.

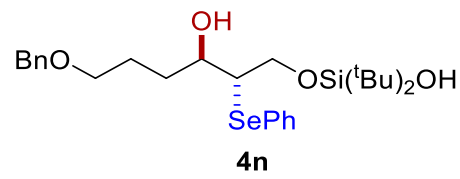

((6-(benzyloxy)-3-hydroxy-2-(phenylselanyl)hexyl)oxy)di-tert-butylsilanol

Compound 4n: Synthesized using the general procedure; Purified using a gradient of 0 to $40 \%$ EtOAc/hexanes on Florisil; single diastereomer; (colorless oil, $37 \mathrm{mg}, 72 \%$ yield); $\left.{ }^{1} \mathrm{H} \mathrm{NMR} \mathrm{(400} \mathrm{MHz,} \mathrm{CDCl}_{3}\right) \delta 7.65-7.54(\mathrm{~m}, 2 \mathrm{H})$, $7.41-7.24(\mathrm{~m}, 8 \mathrm{H}), 4.53(\mathrm{~s}, 2 \mathrm{H}), 4.40(\mathrm{dd}, J=11.0,3.6 \mathrm{~Hz}, 1 \mathrm{H}), 4.19-4.09(\mathrm{~m}, 1 \mathrm{H}), 4.00(\mathrm{ddd}, J=9.2,6.3,3.0 \mathrm{~Hz}$, $1 \mathrm{H}), 3.58-3.45(\mathrm{~m}, 2 \mathrm{H}), 3.32(\mathrm{td}, J=6.1,3.6 \mathrm{~Hz}, 1 \mathrm{H}), 1.97(\mathrm{dddd}, J=13.6,8.3,6.7,3.1 \mathrm{~Hz}, 1 \mathrm{H}), 1.87-1.77(\mathrm{~m}$, 1H), $1.77-1.68(\mathrm{~m}, 1 \mathrm{H}), 1.68-1.50(\mathrm{~m}, 1 \mathrm{H}), 1.05(\mathrm{~s}, 9 \mathrm{H}), 1.04(\mathrm{~s}, 9 \mathrm{H}) .{ }^{13} \mathrm{C}\left\{{ }^{1} \mathrm{H}\right\}$ NMR $\left(100 \mathrm{MHz}, \mathrm{CDCl}_{3}\right) \delta 138.1$, 134.3, 129.4, 129.1, 128.4, 127.7, 127.65, 127.60, 72.9, 72.4, 70.1, 64.5, 54.5, 31.8, 27.6, 27.5, 26.1, 20.6, 20.5. IR 3397, 2934, 2866, 1473, 1101, $833 \mathrm{~cm}^{-1}$.; HRMS (APCI) m/z: [M- $\mathrm{H}_{2} \mathrm{O}+\mathrm{H}^{+}$] calculated for $\mathrm{C}_{27} \mathrm{H}_{41} \mathrm{O}_{3} \mathrm{SeSi} 521.1985$, found 521.2015. 
di-tert-butyl((3-hydroxy-6-phenoxy-2-(phenylselanyl)hexyl)oxy)silanol

Compound 4o: Synthesized using the general procedure; Purified using a gradient of 0 to $30 \%$ EtOAc/hexanes on Florisil; single diastereomer; (colorless oil, $38 \mathrm{mg}, 76 \%$ yield); ${ }^{1} \mathrm{H} \mathrm{NMR}\left(400 \mathrm{MHz}, \mathrm{CDCl}_{3}\right) \delta 7.64-7.57$ (m, 2H), $7.34-7.25$ (m, 5H), $6.97(\mathrm{tt}, J=7.3,1.1 \mathrm{~Hz}, 1 \mathrm{H}), 6.94-6.87(\mathrm{~m}, 2 \mathrm{H}), 4.42(\mathrm{dd}, J=11.1,3.7 \mathrm{~Hz}, 1 \mathrm{H}), 4.22-4.11$ (m, 1H), 4.02 (dddd, $J=14.6,12.3,6.8,4.0 \mathrm{~Hz}, 3 \mathrm{H}), 3.34(\mathrm{td}, J=6.2,3.6 \mathrm{~Hz}, 1 \mathrm{H}), 2.03(\mathrm{ddt}, J=13.1,8.8,2.6 \mathrm{~Hz}$, 2H), $1.99-1.85(\mathrm{~m}, 1 \mathrm{H}), 1.74-1.59(\mathrm{~m}, 1 \mathrm{H}), 1.05(\mathrm{~s}, 9 \mathrm{H}), 1.04(\mathrm{~s}, 9 \mathrm{H}) .{ }^{13} \mathrm{C}\left\{{ }^{1} \mathrm{H}\right\} \mathrm{NMR}\left(100 \mathrm{MHz}, \mathrm{CDCl}_{3}\right) \delta 158.9$, 134.4, 129.4, 129.2, 129.1, 127.7, 120.6, 114.5, 72.6, 67.6, 64.8, 54.2, 31.5, 27.5, 27.4, 25.7, 20.6, 20.5. IR 3397, 2934, 2860, 1495, 1238, $827 \mathrm{~cm}^{-1}$; HRMS (APCI) m/z: [M- $\left.\mathrm{H}_{2} \mathrm{O}+\mathrm{H}^{+}\right]$calculated for $\mathrm{C}_{26} \mathrm{H}_{39} \mathrm{O}_{3} \mathrm{SeSi} 507.1828$, found 507.1837.

di-tert-butyl(3-hydroxy-3-(2-methoxyphenyl)-2-(phenylselanyl)propoxy)silanol

$4 p$

Compound 4p: Synthesized using the general procedure; Purified using a gradient of 0 to $30 \% \mathrm{EtOAc/hexanes}$ on Florisil; single diastereomer; (colorless oil, $36 \mathrm{mg}, 72 \%$ yield); ${ }^{1} \mathrm{H} \mathrm{NMR}\left(400 \mathrm{MHz}, \mathrm{CDCl}_{3}\right) \delta 7.42$ (dd, $J=7.5,1.6$ $\mathrm{Hz}, 1 \mathrm{H}), 7.39-7.34(\mathrm{~m}, 2 \mathrm{H}), 7.23-7.12(\mathrm{~m}, 4 \mathrm{H}), 6.95(\mathrm{td}, J=7.5,1.0 \mathrm{~Hz}, 1 \mathrm{H}), 6.72-6.65(\mathrm{~m}, 1 \mathrm{H}), 5.22(\mathrm{~d}, J=6.5$ $\mathrm{Hz}, 1 \mathrm{H}), 4.41(\mathrm{dd}, J=10.9,2.6 \mathrm{~Hz}, 1 \mathrm{H}), 4.16(\mathrm{dd}, J=10.9,3.5 \mathrm{~Hz}, 1 \mathrm{H}), 3.75-3.69(\mathrm{~m}, 1 \mathrm{H}), 3.61(\mathrm{~s}, 3 \mathrm{H}), 1.08$ (s, 9H), 1.06 (s, 9H). ${ }^{13} \mathrm{C}\left\{{ }^{1} \mathrm{H}\right\} \mathrm{NMR}\left(100 \mathrm{MHz}, \mathrm{CDCl}_{3}\right) \delta 156.5,134.5,130.0,129.6,128.8,128.74,128.72,127.2,120.6$, 110.4, 73.6, 64.4, 55.0, 52.7, 27.7, 27.6, 20.8, 20.7. IR 3425, 2929, 2854, 1490, 1467, 1438, 1238, 1107, 1073, 1027, 827, $742 \mathrm{~cm}^{-1}$; HRMS (APCI) m/z: [M- $\left.\mathrm{H}_{2} \mathrm{O}+\mathrm{H}^{+}\right]$calculated for $\mathrm{C}_{24} \mathrm{H}_{35} \mathrm{O}_{3} \mathrm{SeSi} 479.1515$, found 479.1516 .

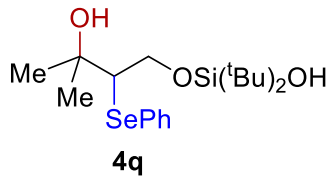

di-tert-butyl(3-hydroxy-3-methyl-2-(phenylselanyl)butoxy)silanol

Compound 4q: Synthesized using the general procedure; Purified using a gradient of 0 to $30 \%$ EtOAc/hexanes on Florisil; (white solid, $31 \mathrm{mg}, 78 \%$ yield); ${ }^{1} \mathrm{H}$ NMR (400 MHz, $\left.\mathrm{CDCl}_{3}\right) \delta 7.65-7.54(\mathrm{~m}, 2 \mathrm{H}), 7.32-7.21$ (m, 3H), $4.35(\mathrm{dd}, J=11.3,3.8 \mathrm{~Hz}, 1 \mathrm{H}), 4.25(\mathrm{dd}, J=11.3,6.7 \mathrm{~Hz}, 1 \mathrm{H}), 3.26(\mathrm{dd}, J=6.7,3.8 \mathrm{~Hz}, 1 \mathrm{H}), 1.45(\mathrm{~s}, 3 \mathrm{H}), 1.43(\mathrm{~s}$, 3H), 1.04 (s, 9H), $1.00(\mathrm{~s}, 9 \mathrm{H}) .{ }^{13} \mathrm{C}\left\{{ }^{1} \mathrm{H}\right\} \operatorname{NMR}\left(100 \mathrm{MHz}, \mathrm{CDCl}_{3}\right) \delta 134.2,130.2,129.3,127.7,73.7,66.0,59.7,29.3$, 
27.5, 20.6, 20.5. IR 3391, 2934, 2860, 1473, $1072 \mathrm{~cm}^{-1}$.; HRMS (APCI) m/z: [M- $\left.\mathrm{H}_{2} \mathrm{O}+\mathrm{H}^{+}\right]$calculated for $\mathrm{C}_{19} \mathrm{H}_{33} \mathrm{O}_{2} \mathrm{SeSi} 401.1410$, found 401.1427 .

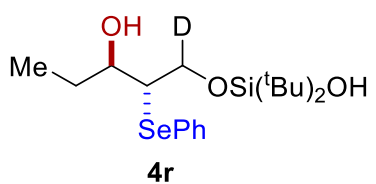

di-tert-butyl((3-hydroxy-2-(phenylselanyl)pentyl-1- $d$ )oxy)silanol

Compound 4r: Synthesized using the general procedure; Purified using a gradient of of 0 to $30 \%$ EtOAc/hexanes on Florisil; 1:1 mixture of diastereomers; (colorless oil, $25 \mathrm{mg}, 62 \%$ yield); ${ }^{1} \mathrm{H}$ NMR (400 MHz, $\mathrm{CDCl}_{3}$ ) $\delta 7.64-7.48$ (m, 4H), $7.33-7.20(\mathrm{~m}, 6 \mathrm{H}), 4.35(\mathrm{~d}, J=3.6 \mathrm{~Hz}, 1 \mathrm{H}), 4.11(\mathrm{dd}, J=10.5,6.6 \mathrm{~Hz}, 1 \mathrm{H}), 3.84$ (ddd, $J=8.6,6.1,3.6 \mathrm{~Hz}$, $2 \mathrm{H}), 3.29(\mathrm{td}, J=5.3,1.4 \mathrm{~Hz}, 2 \mathrm{H}), 1.86-1.66(\mathrm{~m}, 2 \mathrm{H}), 1.63-1.49(\mathrm{~m}, 2 \mathrm{H}), 1.01(\mathrm{~m}, 42 \mathrm{H}) .{ }^{13} \mathrm{C}\left\{{ }^{1} \mathrm{H}\right\} \mathrm{NMR}(125 \mathrm{MHz}$, $\left.\mathrm{CDCl}_{3}\right) \delta 134.4,129.4,129.3,127.8,74.6,64.6(\mathrm{dd}, J=22.3,14.8 \mathrm{~Hz}), 53.9,53.8,28.0,27.6,27.5$, 20.7, 20.6, 10.3. IR 3385, 2934, 2860, 1461, $827 \mathrm{~cm}^{-1}$; HRMS (APCI) m/z: [M + $\left.\mathrm{H}^{+}\right]$calculated for $\mathrm{C}_{19} \mathrm{H}_{32} \mathrm{DO}_{2} \mathrm{SeSi} 402.1472$, found 402.1482 .

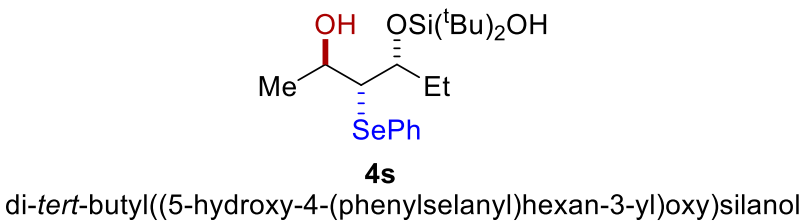

Compound 4s: Synthesized using the general procedure; Purified using a gradient of 0 to $30 \%$ EtOAc/hexanes on Florisil; single diastereomer; (crystalline solid, $16 \mathrm{mg}, 40 \%$ yield); ${ }^{1} \mathrm{H} \mathrm{NMR}\left(400 \mathrm{MHz}, \mathrm{CDCl}_{3}\right) \delta 7.59-7.50(\mathrm{~m}, 2 \mathrm{H})$, $7.32-7.21(\mathrm{~m}, 3 \mathrm{H}), 4.35(\mathrm{ddd}, J=9.4,4.1,1.2 \mathrm{~Hz}, 1 \mathrm{H}), 4.08(\mathrm{p}, J=6.4 \mathrm{~Hz}, 1 \mathrm{H}), 3.12(\mathrm{dd}, J=6.4,1.2 \mathrm{~Hz}, 1 \mathrm{H}), 2.26$ $-2.13(\mathrm{~m}, 1 \mathrm{H}), 1.80-1.72(\mathrm{~m}, 1 \mathrm{H}), 1.35$ (d, $J=6.4 \mathrm{~Hz}, 3 \mathrm{H}), 1.06$ (s, 9H), 1.04 (s, 9H), 0.89 (t, $J=7.5 \mathrm{~Hz}, 3 \mathrm{H})$. ${ }^{13} \mathrm{C}\left\{{ }^{1} \mathrm{H}\right\}$ NMR $\left(100 \mathrm{MHz}, \mathrm{CD}_{3} \mathrm{CN}\right) \delta 134.0,132.4,130.1,127.7,74.0,70.0,61.3,29.9,28.2,28.0,22.8,21.5,21.4$, 10.6.; IR 3334, 2940, 2860, 1473, 1061, $827 \mathrm{~cm}^{-1}$; HRMS (APCI) m/z: $\left[\mathrm{M}-\mathrm{H}_{2} \mathrm{O}+\mathrm{H}^{+}\right]$calculated for $\mathrm{C}_{20} \mathrm{H}_{35} \mathrm{O}_{2} \mathrm{SeSi}$ 415.1566, found 415.1583 .

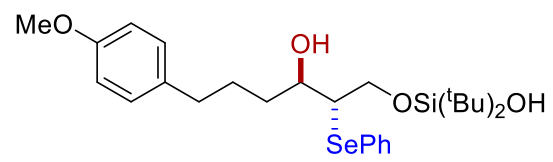

di-tert-butyl((3-hydroxy-6-(4-methoxyphenyl)-2 (phenylselanyl)hexyl)oxy)silanol

Compound 4t: Synthesized using the general procedure; Purified using a gradient of 0 to $30 \%$ EtOAc/hexanes on Florisil; single diastereomer; (colorless oil, $36 \mathrm{mg}, 67 \%$ yield); ${ }^{1} \mathrm{H} \mathrm{NMR}\left(400 \mathrm{MHz}, \mathrm{CDCl}_{3}\right) \delta 7.62-7.52(\mathrm{~m}, 2 \mathrm{H})$, $7.35-7.23(\mathrm{~m}, 3 \mathrm{H}), 7.13-7.06(\mathrm{~m}, 2 \mathrm{H}), 6.88-6.80(\mathrm{~m}, 2 \mathrm{H}), 4.38(\mathrm{dd}, J=11.1,3.7 \mathrm{~Hz}, 1 \mathrm{H}), 4.12(\mathrm{dd}, J=11.1,6.1$ $\mathrm{Hz}, 1 \mathrm{H}), 3.97$ (ddd, $J=8.7,6.0,3.1 \mathrm{~Hz}, 1 \mathrm{H}), 3.81(\mathrm{~s}, 3 \mathrm{H}), 3.29$ (td, $J=6.0,3.6 \mathrm{~Hz}, 1 \mathrm{H}), 2.61-2.51(\mathrm{~m}, 2 \mathrm{H}), 1.89-$ 
$1.71(\mathrm{~m}, 2 \mathrm{H}), 1.71-1.62(\mathrm{~m}, 1 \mathrm{H}), 1.62-1.49(\mathrm{~m}, 1 \mathrm{H}), 1.05(\mathrm{~s}, 9 \mathrm{H}), 1.03(\mathrm{~s}, 9 \mathrm{H}) .{ }^{13} \mathrm{C}\left\{{ }^{1} \mathrm{H}\right\} \mathrm{NMR}\left(100 \mathrm{MHz}^{\mathrm{CDCl}}\right)_{3}$ $\delta 157.7,134.4,129.3,129.23,129.20,127.7,113.7,72.9,64.7,55.2,54.2,34.7,34.4,27.8,27.5,27.4,20.6,20.4$. IR

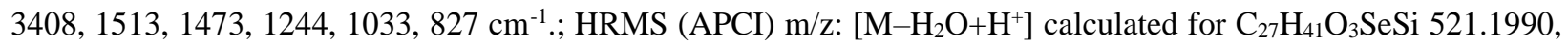
found 521.2012.

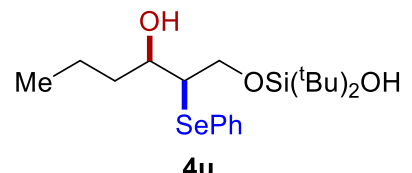

di-tert-butyl((3-hydroxy-2-(phenylselanyl)hexyl)oxy)silanol

Compound 4u: Synthesized using the general procedure; Purified using a gradient of 0 to 30\% EtOAc/hexanes on Florisil; single diastereomer; (colorless oil, $30 \mathrm{mg}, 64 \%$ yield); ${ }^{1} \mathrm{H}$ NMR (400 MHz, $\left.\mathrm{CDCl}_{3}\right) \delta 7.60-7.54(\mathrm{~m}, 2 \mathrm{H})$, $7.29-7.18(\mathrm{~m}, 3 \mathrm{H}), 4.25-4.02(\mathrm{~m}, 3 \mathrm{H}), 3.26(\mathrm{ddd}, J=7.6,4.3,2.6 \mathrm{~Hz}, 1 \mathrm{H}), 1.75(\mathrm{dddd}, J=13.4,9.7,8.1,5.2 \mathrm{~Hz}$, $1 \mathrm{H}), 1.61$ (dddd, $J=13.5,9.5,6.2,5.0 \mathrm{~Hz}, 1 \mathrm{H}), 1.46(\mathrm{dddd}, J=12.8,9.7,7.4,5.4 \mathrm{~Hz}, 1 \mathrm{H}), 1.40-1.27$ (m, $1 \mathrm{H}), 1.02$ $(\mathrm{s}, 9 \mathrm{H}), 1.01(\mathrm{~s}, 9 \mathrm{H}), 0.90(\mathrm{t}, J=7.3 \mathrm{~Hz}, 3 \mathrm{H}) \cdot ;{ }^{13} \mathrm{C}\left\{{ }^{1} \mathrm{H}\right\} \mathrm{NMR}\left(100 \mathrm{MHz}, \mathrm{CDCl}_{3}\right) \delta 134.4,129.6,129.3,127.6,71.6$, 65.9, 55.5, 37.8, 27.7, 27.6, 20.8, 20.6, 19.2, 14.1. IR 3397, 2934, 2860, 1473, $827 \mathrm{~cm}^{-1}$; HRMS (APCI) m/z: [M$\left.\mathrm{H}_{2} \mathrm{O}+\mathrm{H}^{+}\right]$calculated for $\mathrm{C}_{20} \mathrm{H}_{35} \mathrm{O}_{2} \mathrm{SeSi} 415.1566$, found 415.1590 .

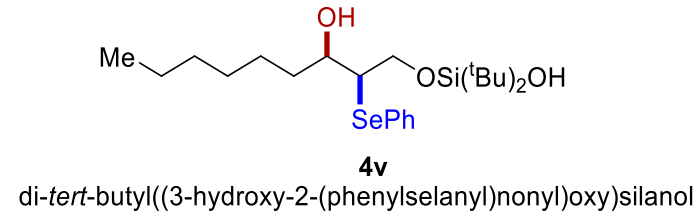

Compound 4v: Synthesized using the general procedure; Purified using a gradient of 0 to $30 \%$ EtOAc/hexanes on Florisil; single diastereomer; (colorless oil, $33 \mathrm{mg}, 70 \%$ yield); ${ }^{1} \mathrm{H} \mathrm{NMR}\left(500 \mathrm{MHz}, \mathrm{CDCl}_{3}\right) \delta 7.55-7.47$ (m, 2H), $7.19(\mathrm{~m}, 3 \mathrm{H}), 4.15(\mathrm{dd}, J=10.8,7.7 \mathrm{~Hz}, 1 \mathrm{H}), 4.10(\mathrm{dd}, J=10.8,4.2 \mathrm{~Hz}, 1 \mathrm{H}), 3.99$ (ddd, $J=7.9,5.3,2.6 \mathrm{~Hz}, 1 \mathrm{H})$, $3.21(\mathrm{dt}, J=7.2,3.1 \mathrm{~Hz}, 1 \mathrm{H}), 1.68(\mathrm{dtd}, J=13.5,8.7,5.0 \mathrm{~Hz}, 1 \mathrm{H}), 1.58(\mathrm{ddt}, J=14.7,10.1,5.1 \mathrm{~Hz}, 1 \mathrm{H}), 1.35(\mathrm{~m}$, $1 \mathrm{H}), 1.28-1.09(\mathrm{~m}, 7 \mathrm{H}), 0.96(\mathrm{~s}, 9 \mathrm{H}), 0.95(\mathrm{~s}, 9 \mathrm{H}), 0.80(\mathrm{t}, J=6.7 \mathrm{~Hz}, 3 \mathrm{H}) .{ }^{13} \mathrm{C}\left\{{ }^{1} \mathrm{H}\right\} \mathrm{NMR}\left(125 \mathrm{MHz}, \mathrm{CDCl}_{3}\right) \delta$ 134.2, 129.4, 129.1, 127.5, 71.8, 65.8, 55.4, 35.6, 31.7, 29.1, 27.6, 27.4, 25.8, 22.6, 20.7, 20.4, 14.0. IR 3397, 2934, 2871, 1473, 1090, $827 \mathrm{~cm}^{-1}$; HRMS (APCI) m/z: [M- $\left.\mathrm{H}_{2} \mathrm{O}+\mathrm{H}^{+}\right]$calculated for $\mathrm{C}_{23} \mathrm{H}_{41} \mathrm{O}_{2} \mathrm{SeSi}$ 457.2041, found 457.2060. 


\section{Derivatization Reactions (Scheme 7)}

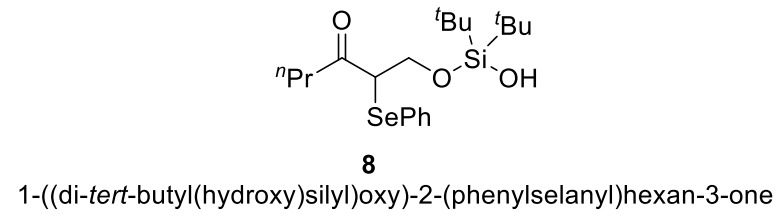

An oven dried tube with a magnetic stir-bar was charged with compound $4 \mathbf{a}\left(0.1 \mathrm{mmol}, 43 \mathrm{mg}, 1.0\right.$ equiv.) and $\mathrm{CH}_{2} \mathrm{Cl}_{2}$ $(2 \mathrm{~mL})$. The solution was cooled to $-10^{\circ} \mathrm{C}$ using a cryogenic cooler, and Dess-Martin periodinane $(0.12 \mathrm{mmol}, 51 \mathrm{mg}$, 1.2 equiv.) was added in one portion. The suspension was stirred for 15 minutes at $-10{ }^{\circ} \mathrm{C}$, and then allowed to warm to $0{ }^{\circ} \mathrm{C}$. After $3 \mathrm{~h}$ at $0{ }^{\circ} \mathrm{C}$, the reaction mixture was diluted with $\mathrm{CH}_{2} \mathrm{Cl}_{2}$ and filtered through a short pad of celite. The filtrate was concentrated under reduced pressure and purified by silica gel column chromatography (gradient of 2-5\% ethyl acetate/hexanes) to afford 8 as a colorless oil (22 mg, $0.051 \mathrm{mmol}, 51 \%$ yield). ${ }^{1} \mathrm{H}$ NMR $\left(400 \mathrm{MHz}, \mathrm{CDCl}_{3}\right) \delta$ $7.58-7.50$ (m, 2H), $7.39-7.27$ (m, 3H), 4.24 (t, $J=10.5 \mathrm{~Hz}, 1 \mathrm{H}), 4.05$ (dd, $J=10.6,4.4 \mathrm{~Hz}, 1 \mathrm{H}), 3.83$ (dd, $J=10.3$, $4.3 \mathrm{~Hz}, 1 \mathrm{H}), 2.79(\mathrm{dt}, J=17.1,7.4 \mathrm{~Hz}, 1 \mathrm{H}), 2.49(\mathrm{dt}, J=17.2,7.1 \mathrm{~Hz}, 1 \mathrm{H}), 1.70-1.53(\mathrm{~m}, 2 \mathrm{H}), 1.01-0.87(\mathrm{~m}, 21 \mathrm{H})$. ${ }^{13} \mathrm{C}\left\{{ }^{1} \mathrm{H}\right\} \mathrm{NMR}\left(100 \mathrm{MHz}, \mathrm{CDCl}_{3}\right) \delta 207.0,135.5,129.4,129.0,127.2,62.8,51.6,43.8,27.6,27.3,20.8,20.3,17.4$, 13.9. IR 3499, 2963, 2934, 2860, 1695, 1473, 1364, 1107, 1084, 1016, 856, 827, 742, 690, $650 \mathrm{~cm}^{-1}$.; HRMS (APCI) $\mathrm{m} / \mathrm{z}:\left[\mathrm{M}+\mathrm{H}^{+}\right]$calculated for $\mathrm{C}_{20} \mathrm{H}_{35} \mathrm{O}_{3} \mathrm{SeSi} 431.1521$, found 431.1507 .

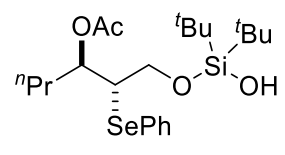

9

1-((di-tert-butyl(hydroxy)silyl)oxy)-2-(phenylselanyl)hexan-3-yl acetate

A solution of compound $4 \mathbf{a}(0.1 \mathrm{mmol}, 43 \mathrm{mg}, 1$ equiv. $)$ in $\mathrm{CH}_{2} \mathrm{Cl}_{2}(1 \mathrm{~mL})$ was cooled to $0{ }^{\circ} \mathrm{C}$ using an ice-water bath. Pyridine (1.0 mmol, $82 \mu \mathrm{L}, 10$ equiv.), acetic anhydride ( $0.5 \mathrm{mmol}, 47 \mu \mathrm{L}, 5$ equiv.) and DMAP (catalytic, $\sim 1.0 \mathrm{mg}$ ) were sequentially added. The mixture was stirred at $0{ }^{\circ} \mathrm{C}$ for $3 \mathrm{~h}$. Next, the reaction mixture was diluted with $5 \mathrm{~mL}$ $\mathrm{CH}_{2} \mathrm{Cl}_{2}$, transferred to a separatory funnel, and washed with water. The organic layer was collected and concentrated after drying over $\mathrm{Na}_{2} \mathrm{SO}_{4}$. The residue was purified by silica gel column chromatography (gradient of 2-5\% ethyl acetate/hexanes) to afford 9 (colorless oil, $46 \mathrm{mg}, 0.096 \mathrm{mmol}, 96 \%$ yield). ${ }^{1} \mathrm{H} \mathrm{NMR}$ (400 MHz, $\left.\mathrm{CDCl}_{3}\right) \delta 7.52-7.46$ (m, 2H), $7.21-7.14$ (m, 3H), 5.25 (ddd, $J=9.1,5.6,3.2 \mathrm{~Hz}, 1 \mathrm{H}), 4.08$ (dd, $J=11.1,4.2 \mathrm{~Hz}, 1 \mathrm{H}), 3.96$ (dd, $J=11.0$, $7.4 \mathrm{~Hz}, 1 \mathrm{H}), 3.46$ (ddd, $J=7.3,5.6,4.3 \mathrm{~Hz}, 1 \mathrm{H}), 1.89$ (s, 3H), $1.75-1.54(\mathrm{~m}, 2 \mathrm{H}), 1.33-1.14(\mathrm{~m}, 2 \mathrm{H}), 0.95(\mathrm{~s}, 9 \mathrm{H})$, $0.93(\mathrm{~s}, 9 \mathrm{H}), 0.80(\mathrm{t}, J=7.4 \mathrm{~Hz}, 3 \mathrm{H}) .{ }^{13} \mathrm{C}\left\{{ }^{1} \mathrm{H}\right\} \mathrm{NMR}\left(100 \mathrm{MHz}, \mathrm{CDCl}_{3}\right) \delta 171.4,134.4,129.6,129.2,127.7,74.2$, 64.2, 52.0, 33.4, 27.7, 27.5, 21.1, 20.6, 20.5, 18.9, 13.8. IR 3505, 2963, 2934, 2860, 1712, 1473, 1370, 1256, 1101, $1022,827,742,690,645 \mathrm{~cm}^{-1}$; HRMS (APCI) $\left[\mathrm{M}^{+}\right]$calculated for $\mathrm{C}_{22} \mathrm{H}_{38} \mathrm{O}_{4} \mathrm{SeSi} 474.1705$, found 474.1702 .

\section{Associated Content}

Copies of ${ }^{1} \mathrm{H}$ and ${ }^{13} \mathrm{C}$ NMR spectra of new compounds, crystallographic data, further experimental details 


\section{Author Information}

*Corresponding Author E-mail: ssathyam@ku.edu.

\section{Acknowledgements}

This work was supported by a National Institutes of Health grant R35GM142499 awarded to Shyam Sathyamoorthi. We gratefully acknowledge Dr. Allen Oliver (University of Notre Dame) for X-ray crystallography analysis.

\section{References}

1. Kočovský, P. Addition Reactions. In Organic Reaction Mechanisms · 2016, Vol 52; Knipe, A.C., Ed.; Wiley \& Sons, 2020; pp 463-659.

2. Slebocka-Tilk, H.; Ball, R. G.; Brown, R. S. The Question of Reversible Formation of Bromonium Ions during the Course of Electrophilic Bromination of Olefins. 2. The Crystal and Molecular Structure of the Bromonium Ion of Adamantylideneadamantane. J. Am. Chem. Soc. 1985, 107, 4504-4508.

3. Overman, L. E.; Campbell, C. B. Hemiacetal Mediated Reactions. Directed Synthesis of Diols and Acetals. J. Org. Chem. 1974, 39, 1474-1481.

4. Sarraf, S. T.; Leighton, J. L. Oxymercuration of Homoallylic Alcohol Derived Hemiacetals: Diastereoselective Synthesis of Protected 1,3-Diols. Org. Lett. 2000, 2, 403-405.

5. Hornberger, K. R.; Hamblett, C. L.; Leighton, J. L. Total Synthesis of Leucascandrolide A. J. Am. Chem. Soc. 2000, 122, 12894-12895.

6. Thomas, A. A.; Nagamalla, S.; Sathyamoorthi, S. Salient Features of the Aza-Wacker Cyclization Reaction. Chem. Sci. 2020, 11, 8073-8088.

7. Parker, P. D.; Lemercier, B. C.; Pierce, J. G. Synthesis of Quaternary-Substituted Thiazolines via Halocyclization of S-Allyl Thioimidate Salts. J. Org. Chem. 2018, 83, 12-22.

8. Corey, E.; Hopkins, P.; Munroe, J.; Marfat, A.; Hashimoto, S.-i. Total Synthesis of 6-Trans, 10-cis and (plus or minus) -6-Trans,8-cis Isomers of Leukotriene B. J. Am. Chem. Soc. 1980, 102, 7986-7987.

9. Myers, A. G.; Widdowson, K. L. Direct Transformation of 2,3-Epoxy Alcohols into Hydroxy Carbonates under Mildly Basic Conditions. Tetrahedron Lett. 1988, 29, 6389-6392. 
10. Trost, B. M.; Sudhakar, A. R. Cis Hydroxyamination Equivalent. Application to the Synthesis of (-)Acosamine. J. Am. Chem. Soc. 1987, 109, 3792-3794.

11. Dhokale, R. A.; Seidl, F. J.; Sathyamoorthi, S. A Formal Rearrangement of Allylic Silanols. Molecules 2021, $26,3829$.

12. Dhokale, R. A.; Seidl, F. J.; Shinde, A. H.; Mague, J. T.; Sathyamoorthi, S. Tethered Silanoxyiodination of Alkenes. J. Org. Chem. 2021, 86, 9233-9243.

13. Shinde, A. H.; Sathyamoorthi, S. Tethered Silanoxymercuration of Allylic Alcohols. Org. Lett. 2020, 22, 8665-8669.

14. Nagamalla, S.; Dhokale, R. A.; Seidl, F. J.; Mague, J. T.; Sathyamoorthi, S. Unusual RearrangementRemercuration Reactions of Allylic Silanols. Org. Chem. Front. 2021, 8, 5361-5368.

15. Santi, C.; Santoro, S. Electrophilic Selenium. In Organoselenium Chemistry: Synthesis and Reactions; Wirth, T., Ed.; Wiley-VCH: Weinheim, Germany, 2012; pp 1-51.

16. Wirth, T. Organoselenium Chemistry in Stereoselective Reactions. Angew. Chem., Int. Ed. 2000, 39, 37403749.

17. Singh, F. V.; Wirth, THY Selenium and Tellurium Electrophiles in Organic Synthesis. Phys. Sci. Rev. 2019, 4.

18. Bowman, W. R. Selenium Compounds in Radical Reactions. In Organoselenium Chemistry: Synthesis and Reactions; Wirth, T., Ed.; Wiley-VCH: Weinheim, Germany, 2012; pp 111-146.

19. Denmark, S. E.; Edwards, M. G. On the Mechanism of the Selenolactonization Reaction with Selenenyl Halides. J. Org. Chem. 2006, 71, 7293-7306.

20. Petragnani, N.; Stefani, H. A.; Valduga, C. J. Recent Advances in Selenocyclofunctionalization Reactions. Tetrahedron 2001, 57, 1411-1448.

21. Denmark, S. E.; Kalyani, D.; Collins, W. R. Preparative and Mechanistic Studies toward the Rational Development of Catalytic, Enantioselective Selenoetherification Reactions. J. Am. Chem. Soc. 2010, 132, 1575215765 .

22. Tiecco, M. Electrophilic Selenium, Selenocyclizations. In Organoselenium Chemistry: Modern Developments in Organic Synthesis; Wirth, T., Ed.; Springer Berlin Heidelberg: Berlin, Heidelberg, 2000 ; pp 7-54. 
23. Lipshutz, B. H.; Gross, T. (2,4,6-Triisopropylphenyl)selenium Bromide (TIPPSe-Br). An in Situ-Generated Reagent for Effecting Highly Selective Ring Closures of Homoallylic Alcohols to Substituted Tetrahydrofurans. J. Org. Chem. 1995, 60, 3572-3573.

24. Reich, H. J. Organoselenium Chemistry. Benzeneselenenyl Trifluoroacetate Additions to Olefins and Acetylenes. J. Org. Chem. 1974, 39, 428-429.

25. Sharpless, K. B.; Lauer, R. F. Electrophilic Organoselenium Reagents. New Route to Allylic Acetates and Ethers. J. Org. Chem. 1974, 39, 429-430.

26. Nicolaou, K. C.; Claremon, D. A.; Barnette, W. E.; Seitz, S. P. $N$-Phenylselenophthalimide $(N$-PSP) and $N$ phenylselenosuccinimide (N-PSS). Two Versatile Carriers of the Phenylseleno Group. Oxyselenation of Olefins and a Selenium-based Macrolide Synthesis. J. Am. Chem. Soc. 1979, 101, 3704-3706.

27. Grieco, P. A.; Jaw, J. Y.; Claremon, D. A.; Nicolaou, K. C. N-Phenylselenophthalimide. A Useful Reagent for the Facile Transformation of (1) Carboxylic Acids into Either Selenol Esters or Amides and (2) Alcohols into Alkyl Phenyl Selenides. J. Org. Chem. 1981, 46, 1215-1217.

28. Cooper, M. A.; Ward, A. D. Formation of Dihydroxyselenides from Allylic Alcohols and Their Conversion to $\beta$-Hydroxy Epoxides via Substitution of a Phenylselenonyl Group. Tetrahedron 2004, 60, 7963-7972.

29. Cooper, M. A.; Ward, A. D., Hydroxyselenation of Allylic Alcohols. Tetrahedron Lett. 1995, 36, 2327-2330.

30. Liotta, D., New Organoselenium Methodology. Acc. Chem. Res. 1984, 17, 28-34. 